\title{
Copper and ligand free Sonogashira reaction catalyzed by Pd(0) nanoparticles at ambient conditions under ultrasound irradiation
}

\author{
Atul R. Gholap, ${ }^{\dagger}$ K. Venkatesan, ${ }^{\dagger}$ Renu Pasricha, ${ }^{\ddagger}$ Thomas Daniel, $^{\dagger}$ R. J. Lahoti, ${ }^{\dagger}$ \\ and K. V. Srinivasan*t \\ ${ }^{\dagger}$ Organic Chemistry; Technology Division, ${ }^{\ddagger}$ Centre for Material Characterization, \\ National Chemical Laboratory, Dr. Homi Bhabha Road, Pune - 411 008, India. \\ E-mail address: kvsri@dalton.ncl.res.in
}

\section{SUPPORTING INFORMATION}

TABLE OF CONTENTS

Contents

1) General considerations and experimental procedure

2) Characterization data

3) Scanned Spectra

4) References for known compounds
Page number

S2-S6

S7-S10

S11-S38

S39 


\section{General Considerations}

All sonochemical reactions were carried out in an argon atmosphere. All of the ionic liquids were prepared by the method reported by us. ${ }^{1}$ All chemicals were of research grade and used without further purification. The reactions were carried out in a thermostated $\left(30 \pm 1{ }^{\circ} \mathrm{C}\right)$ ultrasonic cleaning bath at $50 \mathrm{kHz}$. The ultrasonic cleaner had an output power of $120 \mathrm{~W}$ and a power supply of $450 \mathrm{~W}$. The tank dimensions were 290 $\times 240 \times 150 \mathrm{~mm}$ with a liquid holding capacity of $9.5 \mathrm{~L}$. The reactions were carried out in a RB flask of $10 \mathrm{~mL}$ capacity suspended at the centre of the cleaning bath, $5 \mathrm{~cm}$ below the surface of the liquid. The melting points of the products were uncorrected and compared with the reported literature values. The TEM analysis was carried out in a Transmission Electron Microscope operating at $100 \mathrm{kV}$ at different magnifications varying from 100 to $300 \mathrm{k}$.

\section{Sample Preparation and TEM analysis}

After completion of the reaction a sample is removed from the reaction mixture and after appropriate dilution with isopropyl alcohol was directly deposited on carbon coated 400 mesh $\mathrm{Cu}$ TEM grids. TEM pictures of each sample were taken at $100 \mathrm{kV}$ operating voltage at multiple random locations in the sample and at two different magnifications. The size distribution histogram was obtained on the basis of about 100 particles. 


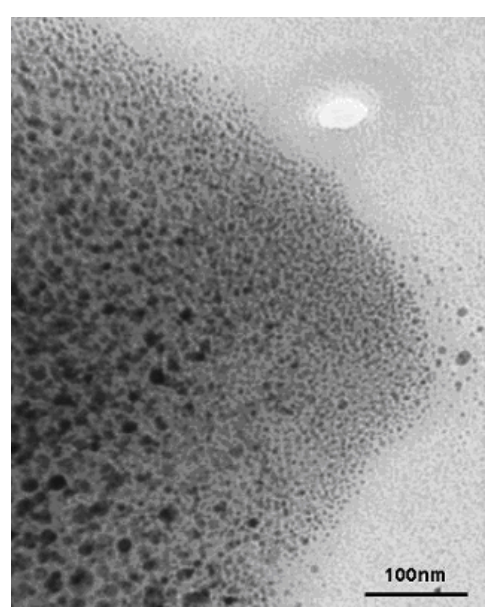

Fig. 1

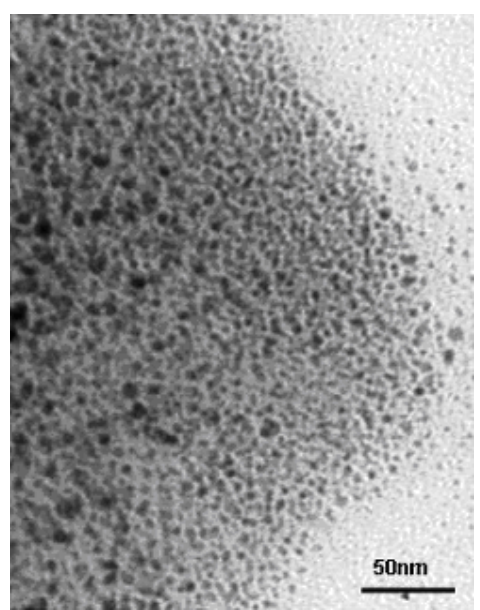

Fig. 2

TEM images of $P d(0)$ nanoparticles in acetone medium at different magnifications

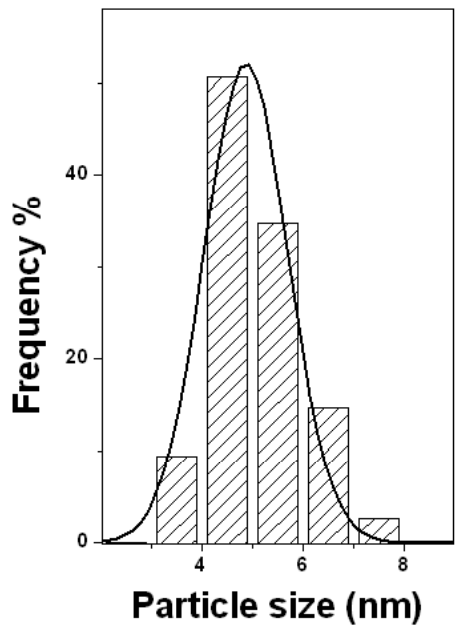

Fig. 3

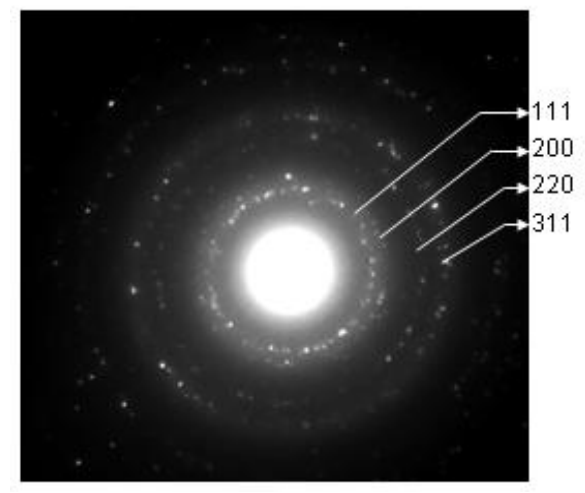

Fig. 4

Histogram and SAED pattern of Pd(0) nanoparticles in acetone medium

The micrograph shows the presence of polydispersed $\operatorname{Pd}(0)$ nanoparticles varying in size from 3-8 $\mathrm{nm}$ and of irregular morphology and spherical in shape (Figs. 1 and 2). The average size of the grains obtained from the TEM picture from acetone medium is about 4.18 nm (Fig. 3). 
The inset picture of Fig. 4 show the SAED (Selected Area Electron Diffraction) pattern of the palladium nanoparticles in the acetone medium. It is clear from SAED pattern that the Pd particles are polycrystalline in nature and it can be indexed as the (111), (200), (220), (311) allowed reflections from face-centered cubic (fcc) palladium.

\section{TEM Measurements in ionic liquid medium}

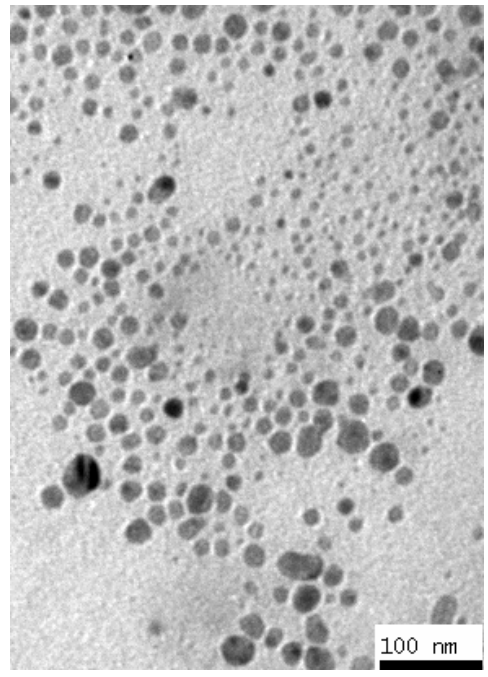

Fig. 5

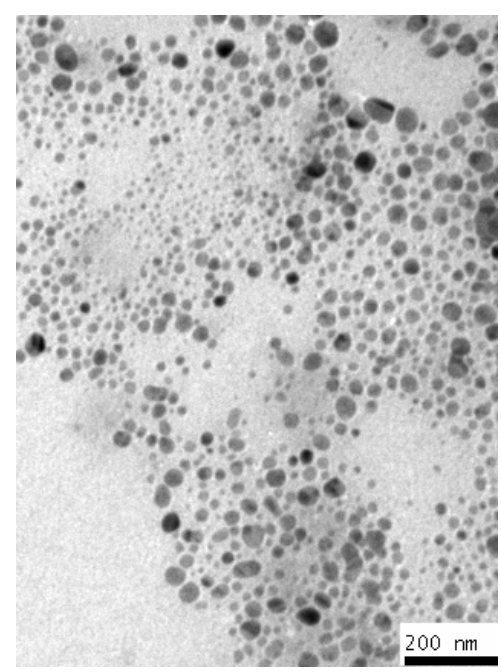

Fig. 6

TEM images of Pd(0) nanoparticles in ionic liquid medium at different magnifications

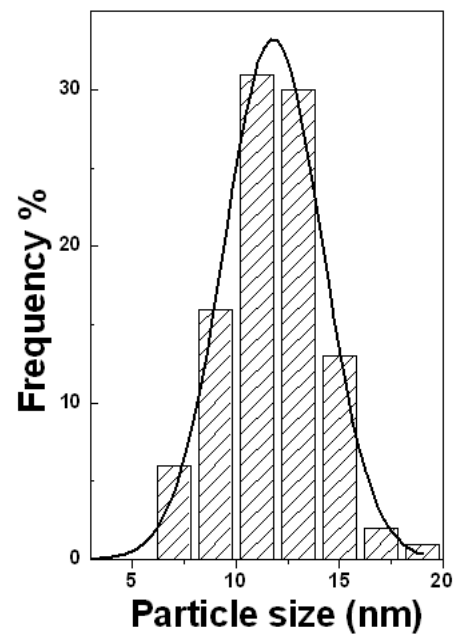

Fig. 7

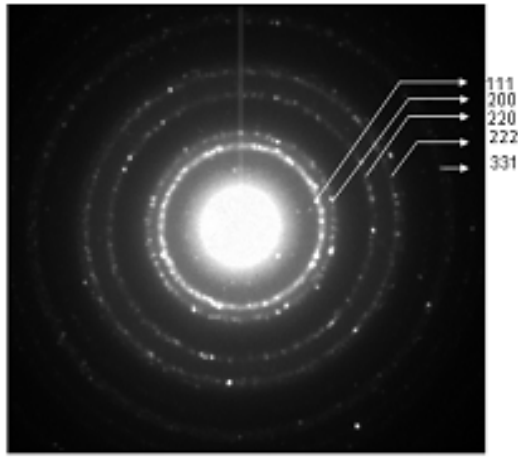

Fig. 8

Histogram and SAED pattern of Pd(0) nanoparticles in IL medium 
In the TEM images (Figs. 5 and 6) obtained from the ionic liquid medium, after the reaction of 1-ethynylbenzene with iodobenzene, it is observed that the palladium nanoparticles are almost spherical in nature and the boundaries are clear. They are well dispersed and the histogram (Fig. 7) shows the size to be in the range of 10-13 nm. The SAED pattern (Fig. 8) indicates the polycrystalline nature of the nanoparticles and can be indexed as the (111), (200), (220), (222), (331) allowed reflections from the fcc palladium.

\section{Experimental}

\section{Preparation of Acetylene Derivatives}

General Procedure for the Sonogashira Reaction in Acetone medium

A mixture of 4-iodotoluene (1 mmol), 1-ethynylbenzene (1 mmol), $\mathrm{PdCl}_{2}(0.02$ $\mathrm{mmol})$ and triethylamine $(1.5 \mathrm{mmol})$ in $5 \mathrm{~mL}$ of acetone was sonicated for the appropriate time under argon at $30{ }^{\circ} \mathrm{C}$. The reaction was monitored by TLC, and after completion of reaction, acetone was removed from reaction mixture under reduced pressure. The residue was dissolved in $10 \%$ ethyl acetate in petroleum ether (bp $60-80{ }^{\circ} \mathrm{C}$ ), clarified by filtration through Celite filter bed, and washed with water. The organic layer was separated and dried over anhyd $\mathrm{Na}_{2} \mathrm{SO}_{4}$ and concentrated in a vacuum. The crude product obtained was further purified by column chromatography through a column of silica gel using petroleum ether (bp $60-80{ }^{\circ} \mathrm{C}$ ) or an appropriate mixture of petroleum ether and ethyl acetate as eluent to afford the pure product.

General Procedure for the Sonogashira Reaction in [bbim] $B F_{4}$

A mixture of 4-iodotoluene $(1 \mathrm{mmol})$, phenylacetylene $(1 \mathrm{mmol}), \mathrm{PdCl}_{2}(0.02$ mmol), and triethylamine $(1.5 \mathrm{mmol})$ in $2 \mathrm{~mL}$ of dry ionic liquid was sonicated for the 
appropriate time under argon at $30{ }^{\circ} \mathrm{C}$. The reaction was monitored by TLC, and after completion of reaction, the product was selectively extracted with $10 \%$ ethyl acetate in petroleum ether (bp $60-80{ }^{\circ} \mathrm{C}$ ) from the reaction mixture leaving behind the ionic liquid consisting of the Pd catalyst as an immiscible layer. The hydrophobic ionic liquid layer was washed with water to remove the amine hydrochloride salt, and the ionic liquid was dried under reduced pressure at $60{ }^{\circ} \mathrm{C}$ for 30 min and used as such for further reaction. The separated organic layer was dried over anhyd $\mathrm{Na}_{2} \mathrm{SO}_{4}$ and concentrated in a vacuum. The crude product obtained was further purified by column chromatography through a column of silica gel using petroleum ether $\left(\mathrm{bp} 60-80{ }^{\circ} \mathrm{C}\right.$ ) or an appropriate mixture of petroleum ether and ethyl acetate as eluent to afford the pure product. 


\section{Characterization data:}

Diphenylacetylene $^{2}$ (Table 1, entry 1): (white solid); mp $60{ }^{\circ} \mathrm{C} ;{ }^{1} \mathrm{H}$ NMR $\left(\mathrm{CDCl}_{3}, 200\right.$ MHz) $\delta$ 7.27-7.24 (m, 6H), 7.48-7.43 (m, 4H); ${ }^{13} \mathrm{C} \mathrm{NMR}\left(\mathrm{CDCl}_{3}, 50 \mathrm{MHz}\right) \delta$ 131.6, 128.3, 128.2, 123.3, 89.4. Anal. Calcd for $\mathrm{C}_{14} \mathrm{H}_{10}$ : C, 94.34; H, 5.66. Found: C, 94.23; H, 5.70 .

1-Fluoro-3-(phenylethynyl)benzene ${ }^{3}$ (Table 1, entry 2): (white solid); mp $36{ }^{\circ} \mathrm{C} ;{ }^{1} \mathrm{H}$ $\mathrm{NMR}\left(\mathrm{CDCl}_{3}, 200 \mathrm{MHz}\right) \delta$ 7.58-7.54 (m, 2H), 7.39-7.31 (m, 5H), 7.27-7.23 (m, 1H), 7.11-7.01 (m, 1H); ${ }^{13} \mathrm{C} \mathrm{NMR}\left(\mathrm{CDCl}_{3}, 50 \mathrm{MHz}\right) \delta 162.3(\mathrm{~d}, J=246.27), 131.62,129.8(\mathrm{~d}$, $J=8.42 \mathrm{~Hz}), 128.4,128.3,127.4(\mathrm{~d}, J=2.56 \mathrm{~Hz}), 125.08(\mathrm{~d}, J=9.52 \mathrm{~Hz}), 122.7,118.3$ (d, $J=23.05 \mathrm{~Hz}), 115.5$ (d, $J=21.22 \mathrm{~Hz}), 90.2,88.0(\mathrm{~d}, J=2.93 \mathrm{~Hz})$. Anal. Calcd for $\mathrm{C}_{14} \mathrm{H}_{9} \mathrm{~F}: \mathrm{C}, 85.70 ; \mathrm{H}, 4.62 ; \mathrm{F}, 9.68$. Found: C, 85.78; H, 4.54; F, 9.75.

4-(phenylethynyl)toluene ${ }^{2}$ (Table 1, entry 3): (white solid); mp $70{ }^{\circ} \mathrm{C} ;{ }^{1} \mathrm{H}$ NMR $\left(\mathrm{CDCl}_{3}, 200 \mathrm{MHz}\right) \delta$ 7.45-7.40 (m, 2H), $7.33(\mathrm{~d}, J=8.22 \mathrm{~Hz}, 2 \mathrm{H}), 7.27-7.21(\mathrm{~m}, 3 \mathrm{H})$, $7.05(\mathrm{~d}, J=8.22 \mathrm{~Hz}, 2 \mathrm{H}), 2.28(\mathrm{~s}, 3 \mathrm{H}) ;{ }^{13} \mathrm{C} \mathrm{NMR}\left(\mathrm{CDCl}_{3}, 50 \mathrm{MHz}\right) \delta 138.3,131.5$, 131.4, 129.0, 128.8, 128.0, 123.4, 120.1, 89.5, 88.7, 21.6. Anal. Calcd for $\mathrm{C}_{15} \mathrm{H}_{12}$ : C, 93.71; H, 6.29. Found: C, 93.70; H, 6.29 .

1-phenylethynylcyclohexanol ${ }^{4}$ (Table 1, entry 4): (white solid); mp $64{ }^{\circ} \mathrm{C} ;{ }^{1} \mathrm{H}$ NMR $\left(\mathrm{CDCl}_{3}, 200 \mathrm{MHz}\right) \delta$ 7.44-7.30 (m, 5H), $2.00(\mathrm{~s}, 1 \mathrm{H}), 1.74-1.43(\mathrm{~m}, 10 \mathrm{H}) ;{ }^{13} \mathrm{C} \mathrm{NMR}$ $\left(\mathrm{CDCl}_{3}, 50 \mathrm{MHz}\right) \delta 131.5,128.0,127.9,122.9,92.9,84.2,68.8,39.9,25.1,23.2$. Calcd. for $\mathrm{C}_{14} \mathrm{H}_{16} \mathrm{O}: \mathrm{C}, 83.96 ; \mathrm{H}, 8.05$. found: $\mathrm{C}, 83.92 ; \mathrm{H}, 8.10$.

1-Fluoro-3-(phenylethynyl)toluene (Table 1, entry 6): (white solid); mp $61{ }^{\circ} \mathrm{C} ;{ }^{1} \mathrm{H}$ NMR $\left(\mathrm{CDCl}_{3}, 200 \mathrm{MHz}\right) \delta 7.36(\mathrm{~d}, J=8.0 \mathrm{~Hz}, 2 \mathrm{H}), 7.25-7.16(\mathrm{~m}, 3 \mathrm{H}), 7.09(\mathrm{~d}, J=7.9$ $\mathrm{Hz}, 2 \mathrm{H}), 7.02-6.90(\mathrm{~m}, 1 \mathrm{H}), 2.30(\mathrm{~s}, 3 \mathrm{H}) ;{ }^{13} \mathrm{C} \mathrm{NMR}\left(\mathrm{CDCl}_{3}, 50 \mathrm{MHz}\right) \delta 162.4(\mathrm{~d}, J=$ $246.47 \mathrm{~Hz}), 138.7,131.5,130.1(\mathrm{~d}, J=9.22 \mathrm{~Hz}), 129.8(\mathrm{~d}, J=9.22 \mathrm{~Hz}), 129.1,128.4$, 127.3, $125.4(\mathrm{~d}, J=11.52 \mathrm{~Hz}), 119.7,119.2(\mathrm{~d}, J=23.03 \mathrm{~Hz}), 118.2(\mathrm{~d}, J=20.73 \mathrm{~Hz})$, $116.8(\mathrm{~d}, J=20.73 \mathrm{~Hz}), 115.3(\mathrm{~d}, J=20.73 \mathrm{~Hz}), 90.5,87.5,21.4$. Anal. Calcd for $\mathrm{C}_{15} \mathrm{H}_{11} \mathrm{~F}: \mathrm{C}, 85.69 ; \mathrm{H}, 5.27$; F, 9.04. Found: C, 85.63; H, 5.32; F, 9.19. 
Di-p-tolylacetylene ${ }^{5}$ (Table 1, entry 7): (white solid); mp $139{ }^{\circ} \mathrm{C} ;{ }^{1} \mathrm{H} \mathrm{NMR}\left(\mathrm{CDCl}_{3}\right.$, $200 \mathrm{MHz}) \delta 7.44(\mathrm{~d}, J=8 \mathrm{~Hz}, 4 \mathrm{H}), 7.17(\mathrm{~d}, J=8 \mathrm{~Hz}, 4 \mathrm{H}), 2.38(\mathrm{~s}, 6 \mathrm{H}) ;{ }^{13} \mathrm{C} \mathrm{NMR}$ $\left(\mathrm{CDCl}_{3}, 50 \mathrm{MHz}\right) \delta 138.1,131.3,129.0,120.3,88.8,21.4$. Anal. Calcd for $\mathrm{C}_{16} \mathrm{H}_{14}: \mathrm{C}$, 93.16; H, 6.84.Found: C, 93.23; H, 6.70.

1-p-Tolylethynylcyclohexanol (Table 1, entry 8): (white solid); mp $103{ }^{\circ} \mathrm{C} ;{ }^{1} \mathrm{H}$ NMR $\left(\mathrm{CDCl}_{3}, 200 \mathrm{MHz}\right) \delta 7.31(\mathrm{~d}, J=8.22 \mathrm{~Hz}, 2 \mathrm{H}), 7.09(\mathrm{~d}, J=8.20 \mathrm{~Hz}, 2 \mathrm{H}), 2.34(\mathrm{~s}, 3 \mathrm{H})$, $1.98(\mathrm{~s}, 1 \mathrm{H}), 1.73-1.57(\mathrm{~m}, 10 \mathrm{H}) ;{ }^{13} \mathrm{C} \mathrm{NMR}\left(\mathrm{CDCl}_{3}, 50 \mathrm{MHz}\right) \delta 138.0,131.5,128.9$, 119.8, 92.1, 84.4, 68.9, 40.0, 25.2, 23.4, 21.4. Anal. Calcd for $\mathrm{C}_{15} \mathrm{H}_{18} \mathrm{O}: \mathrm{C}, 84.07$; $\mathrm{H}$, 8.47. Found: C, 84.03; H, 8.52.

1-Nitro-(4-phenylethynyl)benzene ${ }^{6}$ (Table 1, entry 9): (yellow solid); mp $119{ }^{\circ} \mathrm{C} ;{ }^{1} \mathrm{H}$ $\operatorname{NMR}\left(\mathrm{CDCl}_{3}, 200 \mathrm{MHz}\right) \delta 8.22(\mathrm{~d}, J=8.6 \mathrm{~Hz}, 2 \mathrm{H}), 7.67(\mathrm{~d}, J=8.6 \mathrm{~Hz}, 2 \mathrm{H}), 7.57-7.55$ $(\mathrm{m}, 2 \mathrm{H}), 7.40-7.37(\mathrm{~m}, 3 \mathrm{H}) ;{ }^{13} \mathrm{C} \mathrm{NMR}\left(\mathrm{CDCl}_{3}, 50 \mathrm{MHz}\right) \delta 146.9,132.2,131.8,130.2$, 129.2, 128.5, 123.6, 122.0, 94.6, 87.6. Anal. Calcd for $\mathrm{C}_{14} \mathrm{H}_{9} \mathrm{NO}_{2}$ : C, 75.33; $\mathrm{H}, 4.06$. Found: C, 75.27; H, 4.13.

1-Fluoro-3-(phenylethynyl)nitrobenzene (Table 1, entry 10): (pale yellow solid); mp $139{ }^{\circ} \mathrm{C} ;{ }^{1} \mathrm{H}$ NMR $\left(\mathrm{CDCl}_{3}, 200 \mathrm{MHz}\right) \delta 8.24(\mathrm{~d}, J=8.5 \mathrm{~Hz}, 2 \mathrm{H}), 7.67(\mathrm{~d}, J=8.5 \mathrm{~Hz}, 2 \mathrm{H})$, 7.41-7.08 (m 4H); ${ }^{13} \mathrm{C} \mathrm{NMR}\left(\mathrm{CDCl}_{3}, 50 \mathrm{MHz}\right) \delta 162.3(\mathrm{~d}, J=247.61 \mathrm{~Hz}), 147.1,132.3$, $130.1(\mathrm{~d}, J=9.60 \mathrm{~Hz}), 129.5,127.6(\mathrm{~d}, J=3.83 \mathrm{~Hz}), 123.8(\mathrm{~d}, J=9.60 \mathrm{~Hz}), 123.6,118.5$ $(\mathrm{d}, J=23.03 \mathrm{~Hz}), 116.5,(\mathrm{~d}, J=21.22 \mathrm{~Hz}), 96.09,88.19$. Anal. Calcd for $\mathrm{C}_{14} \mathrm{H}_{8} \mathrm{FNO} 2: \mathrm{C}$, 69.71; H, 3.34; F, 7.88; N, 5.81 Found: C, 69.70; H, 3.40; F, 7.79; N, 5.74.

1-Methyl-4-(phenylethynyl)nitrobenzene (Table 1, entry 11): (yellow solid); mp 157 ${ }^{\circ} \mathrm{C} ;{ }^{1} \mathrm{H} \mathrm{NMR}\left(\mathrm{CDCl}_{3}, 200 \mathrm{MHz}\right) \delta 8.23(\mathrm{~d}, J=8.61 \mathrm{~Hz}, 2 \mathrm{H}), 7.66(\mathrm{~d}, J=7.43 \mathrm{~Hz}, 2 \mathrm{H})$, $7.42(\mathrm{~d}, J=7.44 \mathrm{~Hz}, 2 \mathrm{H}) 7.21(\mathrm{~d}, J=7.82 \mathrm{~Hz}, 2 \mathrm{H}), 2.41(\mathrm{~s}, 3 \mathrm{H}) ;{ }^{13} \mathrm{C} \mathrm{NMR}\left(\mathrm{CDCl}_{3}, 50\right.$ MHz) $\delta 146.7,139.6,132.0,131.6,130.4,129.2$, 123.5, 118.9, 95.0, 87.0, 21.5. Anal. Calcd for $\mathrm{C}_{15} \mathrm{H}_{11} \mathrm{NO}_{2}$ : C, 75.94; H, 4.67; N, 5.90. Found: C, 75.97; H, 4.59; N, 5.82. 
1-(4-Nitro-phenylethynyl)cyclohexanol (Table 1, entry 12): (white solid); mp $101{ }^{\circ} \mathrm{C}$; ${ }^{1} \mathrm{H} \mathrm{NMR}\left(\mathrm{CDCl}_{3}, 200 \mathrm{MHz}\right) \delta 8.15(\mathrm{~d}, J=8.25 \mathrm{~Hz}, 2 \mathrm{H}), 7.54(\mathrm{~d}, J=8.25 \mathrm{~Hz}, 2 \mathrm{H}), 2.01$ (s, $1 \mathrm{H}), 1.76-1.55(\mathrm{~m}, 10 \mathrm{H}) ;{ }^{13} \mathrm{C} \mathrm{NMR}\left(\mathrm{CDCl}_{3}, 50 \mathrm{MHz}\right) \delta 146.9,132.3,129.8,123.4$, 98.3, 82.4, 69.0, 39.7, 25.0, 23.2. Anal. Calcd for $\mathrm{C}_{14} \mathrm{H}_{15} \mathrm{NO}_{3}$ : C, 68.56; H, 6.16; N, 5.71. Found: C, 68.48; H, 6.22; N, 5.68 .

1-Chloro-4-(phenylethynyl)benzene ${ }^{6}$ (Table 1, entry 13): (white solid); mp $83{ }^{\circ} \mathrm{C} ;{ }^{1} \mathrm{H}$ $\mathrm{NMR}\left(\mathrm{CDCl}_{3}, 200 \mathrm{MHz}\right) \delta$ 7.29-7.23 (m, 5H), 7.48-7.37 (m, 4H); ${ }^{13} \mathrm{C} \mathrm{NMR}\left(\mathrm{CDCl}_{3}, 50\right.$ MHz) $\delta 134.2,132.7,131.6,128.6,128.4,128.3,122.9,121.8,90.3$, 88.2. Anal. Calcd for $\mathrm{C}_{14} \mathrm{H}_{9} \mathrm{Cl}$ : C, 79.07; H, 4.27; Cl, 16.67. Found: C, 79.01; H, 4.23; Cl, 16.78.

1-Fluoro-3-(phenylethynyl)chlorobenzene (Table 1, entry 14): (white solid); mp 86 ${ }^{\circ} \mathrm{C} ;{ }^{1} \mathrm{H}$ NMR $\left(\mathrm{CDCl}_{3}, 200 \mathrm{MHz}\right) \delta 7.39(\mathrm{~d}, J=8.33 \mathrm{~Hz}), 7.31-7.11(\mathrm{~m}, 5 \mathrm{H}), 7.03-6.93$ $(\mathrm{m}, 1 \mathrm{H}) ;{ }^{13} \mathrm{C} \mathrm{NMR}\left(\mathrm{CDCl}_{3}, 50 \mathrm{MHz}\right) \delta 162.4(\mathrm{~d}, J=246.48 \mathrm{~Hz}), 134.6,132.8,131.5$, $129.9(\mathrm{~d}, J=6.91 \mathrm{~Hz}), 128.7,128.3,127.4,124.8(\mathrm{~d}, J=9.21 \mathrm{~Hz}), 121.31,118.3(\mathrm{~d}, J=$ $23.03 \mathrm{~Hz}), 115.7(\mathrm{~d}, J=20.73 \mathrm{~Hz}), 89.0(\mathrm{~d}, J=9.22 \mathrm{~Hz})$; Anal. Calcd for $\mathrm{C}_{14} \mathrm{H}_{8} \mathrm{ClF}: \mathrm{C}$, 72.90; H, 3.50; Cl, 15.37; F, 8.24. Found: C, 72.83; H, 3.45; Cl, 15.45; F, 8.19.

1-Chloro-4-(phenylethynyl)toluene ${ }^{7}$ (Table 1, entry 15): (white solid); mp $149{ }^{\circ} \mathrm{C} ;{ }^{1} \mathrm{H}$ NMR $\left(\mathrm{CDCl}_{3}, 200 \mathrm{MHz}\right)$ 7.39-7.32 (m, 4H), 7.26-7.17 (m, 2H), 7.08 (d, $J=7.83,2 \mathrm{H}$ $\mathrm{Hz}), 2.29$ (s, 3H); ${ }^{13} \mathrm{C} \mathrm{NMR}\left(\mathrm{CDCl}_{3}, 50 \mathrm{MHz}\right) \delta 138.6,134.0,132.7,131.5,129.1,128.6$, 122.1, 119.9, 90.5, 87.6, 21.4. Anal. Calcd for $\mathrm{C}_{15} \mathrm{H}_{11} \mathrm{Cl}$ : C, 79.47; $\mathrm{H}, 4.89 ; \mathrm{Cl}, 15.64$. Found: C, 79.42; H, 4.91; Cl, 15.59.

1-(4-Chloro-phenylethynyl)cyclohexanol (Table 1, entry 16): (white solid); mp $78{ }^{\circ} \mathrm{C}$; ${ }^{1} \mathrm{H}$ NMR $\left(\mathrm{CDCl}_{3}, 200 \mathrm{MHz}\right) \delta$ 7.31-7.18 (m, 4H), $1.92(\mathrm{~s}, 1 \mathrm{H}), 1.68-1.49(\mathrm{~m}, 10 \mathrm{H}) ;{ }^{13} \mathrm{C}$ NMR $\left(\mathrm{CDCl}_{3}, 50 \mathrm{MHz}\right) \delta 134.2,132.6,128.5,121.3,93.8,83.8,69.0,39.9,25.1,23.3$. Anal. Calcd for $\mathrm{C}_{14} \mathrm{H}_{15} \mathrm{ClO}$ : C, 71.64; H, 6.44; Cl, 15.10. Found: C, 71.58; H, 6.38; $\mathrm{Cl}$, 15.12 . 
1-(4-Phenylethynyl-phenyl)ethanone ${ }^{2}$ (Table 1, entry 17): (white solid); mp 95-96 ${ }^{\circ} \mathrm{C}$; ${ }^{1} \mathrm{H}$ NMR $\left(\mathrm{CDCl}_{3}, 200 \mathrm{MHz}\right) \delta 7.87(\mathrm{~d}, J=8.72 \mathrm{~Hz}, 2 \mathrm{H}), 7.54(\mathrm{~d}, J=8.59 \mathrm{~Hz}, 2 \mathrm{H}), 7.50$ $7.46(\mathrm{~m}, 2 \mathrm{H}), 7.31-7.28(\mathrm{~m}, 3 \mathrm{H}), 2.54(\mathrm{~s}, 3 \mathrm{H}) ;{ }^{13} \mathrm{C} \mathrm{NMR}\left(\mathrm{CDCl}_{3}, 50 \mathrm{MHz}\right) \delta 197.1$, 136.0, 131.6, 131.5, 128.7, 128.3, 128.1, 128.0, 122.5, 92.6, 88.5, 26.4. Anal. Calcd for $\mathrm{C}_{16} \mathrm{H}_{12} \mathrm{O}: \mathrm{C}, 87.25$; H, 5.49. Found: C, 87.31; H, 5.42.

1-[4-(3'-Fluoro-phenylethynyl)-phenyl]ethanone (Table 1, entry 18): (white solid); mp 90-91 ${ }^{\circ} \mathrm{C} ;{ }^{1} \mathrm{H} \mathrm{NMR}\left(\mathrm{CDCl}_{3}, 200 \mathrm{MHz}\right) \delta 7.87(\mathrm{~d}, J=8.79 \mathrm{~Hz}, 2 \mathrm{H}), 7.53(\mathrm{~d}, J=8.06$ $\mathrm{Hz}, 2 \mathrm{H}), 7.27-6.99(\mathrm{~m}, 4 \mathrm{H}), 2.54$ (s, 3H); ${ }^{13} \mathrm{C} \mathrm{NMR}\left(\mathrm{CDCl}_{3}, 50 \mathrm{MHz}\right) \delta 197.1,162.2(\mathrm{~d}$, $J=247.0 \mathrm{~Hz}), 136.3,131.6,129.9$ (d, $J=8.78 \mathrm{~Hz}), 128.1,127.56,127.50,124.3$ (d, $J=$ $9.52 \mathrm{~Hz}), 118.3(\mathrm{~d}, J=23.05 \mathrm{~Hz}), 116.05$ (d, $J=21.22 \mathrm{~Hz}), 91.1,89.3,26.4$. Anal. Calcd for $\mathrm{C}_{16} \mathrm{H}_{11} \mathrm{FO}$ : C, 80.66; H, 4.65; F, 7.97. Found: C, 80.64; H, 4.66; F, 7.90.

1-(4-p-Tolylethynyl-phenyl)ethanone ${ }^{8}$ (Table 1, entry 19): (white solid); mp 126-127 ${ }^{\circ} \mathrm{C} ;{ }^{1} \mathrm{H} \mathrm{NMR}\left(\mathrm{CDCl}_{3}, 200 \mathrm{MHz}\right) \delta 7.86(\mathrm{~d}, J=8.59 \mathrm{~Hz}, 2 \mathrm{H}), 7.52(\mathrm{~d}, J=8.59 \mathrm{~Hz}, 2 \mathrm{H})$, $7.37(\mathrm{~d}, J=8.08 \mathrm{~Hz}, 2 \mathrm{H}), 7.10(\mathrm{~d}, J=7.83 \mathrm{~Hz}, 2 \mathrm{H}), 2.54(\mathrm{~s}, 3 \mathrm{H}), 2.31(\mathrm{~s}, 3 \mathrm{H}) ;{ }^{13} \mathrm{C}$ $\mathrm{NMR}\left(\mathrm{CDCl}_{3}, 50 \mathrm{MHz}\right) \delta 197.2,139.0,135.9,131.57,131.53,129.1,128.3,128.1$, 119.4, 92.9, 88.0, 26.5, 21.5. Anal. Calcd for $\mathrm{C}_{17} \mathrm{H}_{14} \mathrm{O}$ : C, 87.15; H, 6.02. Found: C, 87.16; H, 5.98.

1-[4-(1'-Hydroxy-cyclohexylethynyl)-phenyl]ethanone (Table 1, entry 20): (white solid); mp 82-83 ${ }^{\circ} \mathrm{C} ;{ }^{1} \mathrm{H}$ NMR $\left(\mathrm{CDCl}_{3}, 200 \mathrm{MHz}\right) \delta 7.88(\mathrm{~d}, J=8.79 \mathrm{~Hz}, 2 \mathrm{H}), 7.48(\mathrm{~d}, J$ $=8.79 \mathrm{~Hz}, 2 \mathrm{H}), 2.58(\mathrm{~s}, 3 \mathrm{H}), 1.99(\mathrm{~s}, 1 \mathrm{H}), 1.7-1.54(\mathrm{~m}, 10 \mathrm{H}) ;{ }^{13} \mathrm{C} \mathrm{NMR}\left(\mathrm{CDCl}_{3}, 50\right.$ MHz) $\delta 197.4,136.0,131.7,128.1,127.8,96.3,83.4,69.0,39.8,26.5,25.0,23.2$. Anal. Calcd for $\mathrm{C}_{16} \mathrm{H}_{18} \mathrm{O}_{2}$ : C, 79.31; H, 7.49. Found: C, 79.35; H, 7.44.

Pd-biscarbene Complex [A] ${ }^{\text {?: }}$ white amorphous powder; ${ }^{1} \mathrm{H} \mathrm{NMR}\left(\mathrm{CDCl}_{3}, 200 \mathrm{MHz}\right) \delta$ $6.81(\mathrm{~s}, 4 \mathrm{H}), 4.52(\mathrm{t}, 8 \mathrm{H}), 2.08(\mathrm{~m}, 8 \mathrm{H}), 1.47(\mathrm{~m}, 8 \mathrm{H}),, 1.01(\mathrm{t}, 12 \mathrm{H}) ;{ }^{13} \mathrm{C} \mathrm{NMR}\left(\mathrm{CDCl}_{3}\right.$, $50 \mathrm{MHz}) \delta 169.2,119.8,50.1,32.6,19.6,13.3 . \mathrm{MS}: \mathrm{m} / z 466\left[\mathrm{M}^{+}-\mathrm{BF}_{4}\right]$. Anal. Calcd for $\mathrm{C}_{12} \mathrm{H}_{24} \mathrm{~N}_{4} \mathrm{Pd}: \mathrm{C}, 43.58 ; \mathrm{H}, 7.31 ; \mathrm{N}, 16.94$. Found: C, 43.55; H, 7.27; N, 16.97. 

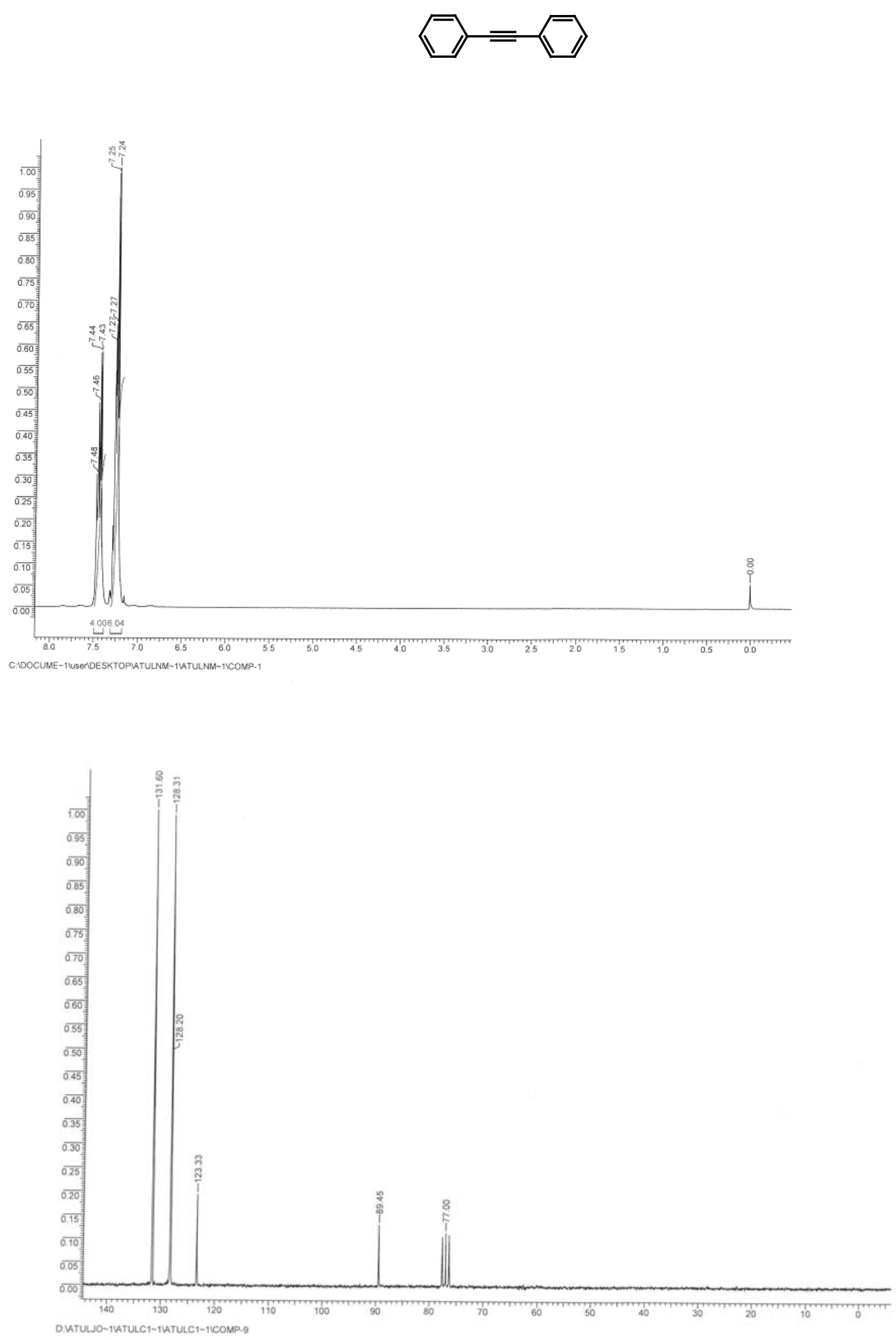

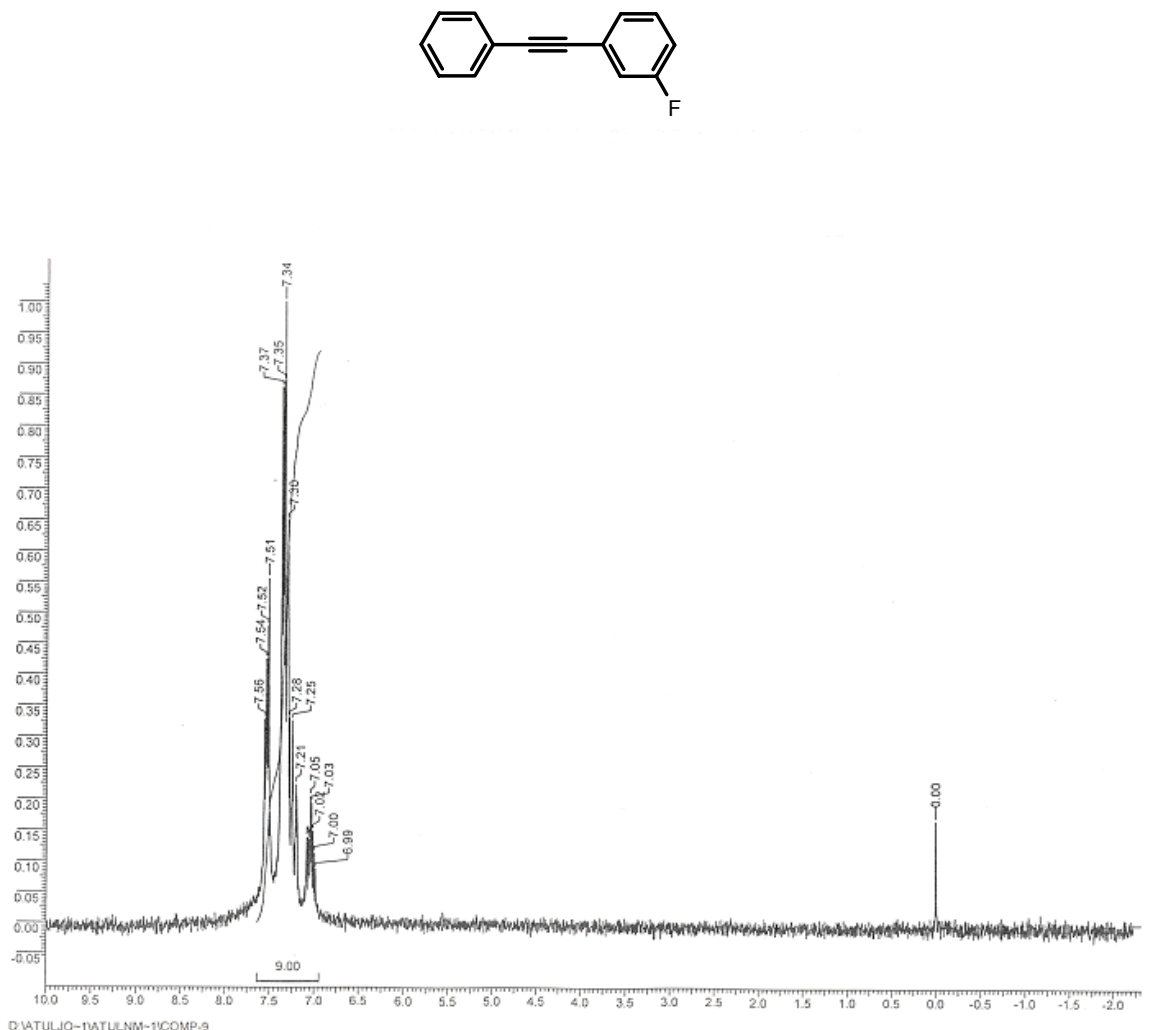
DIATULJO-11ATLENAT-11COMP.9.

Aromatic region (Entry 2)

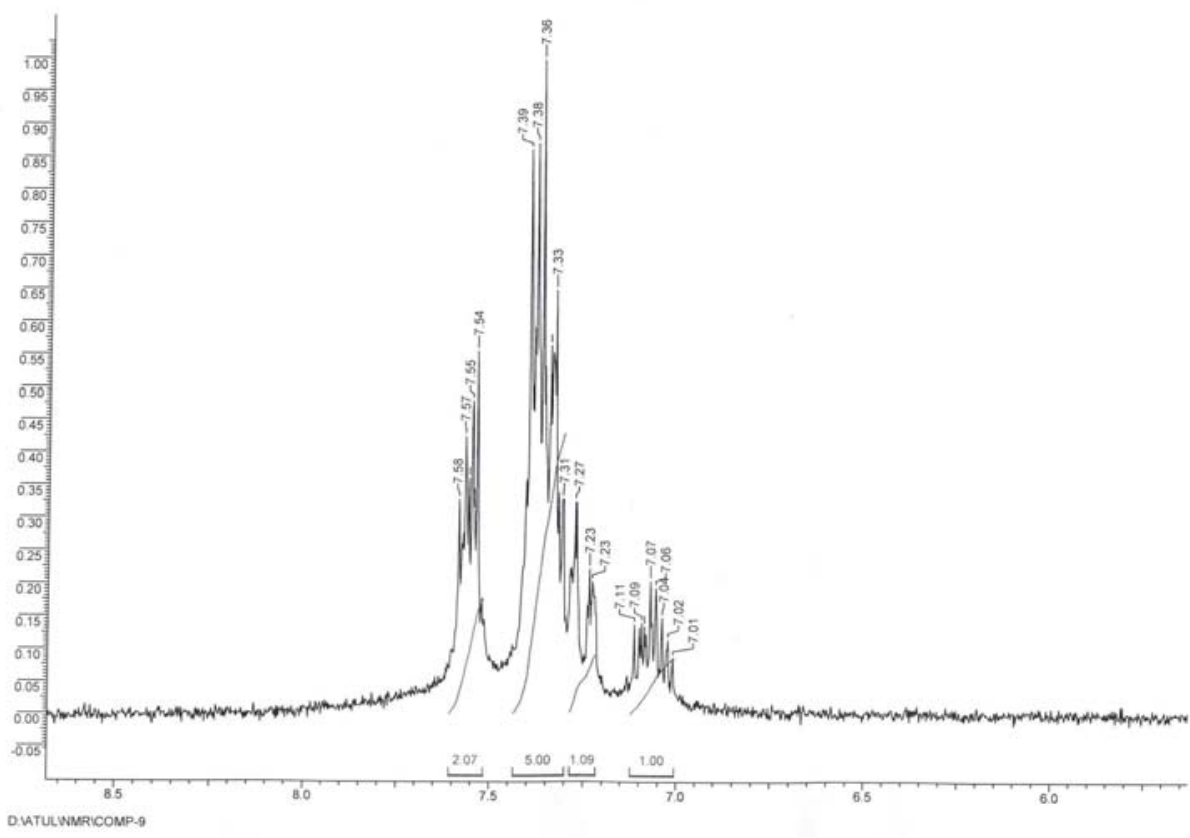

S12 

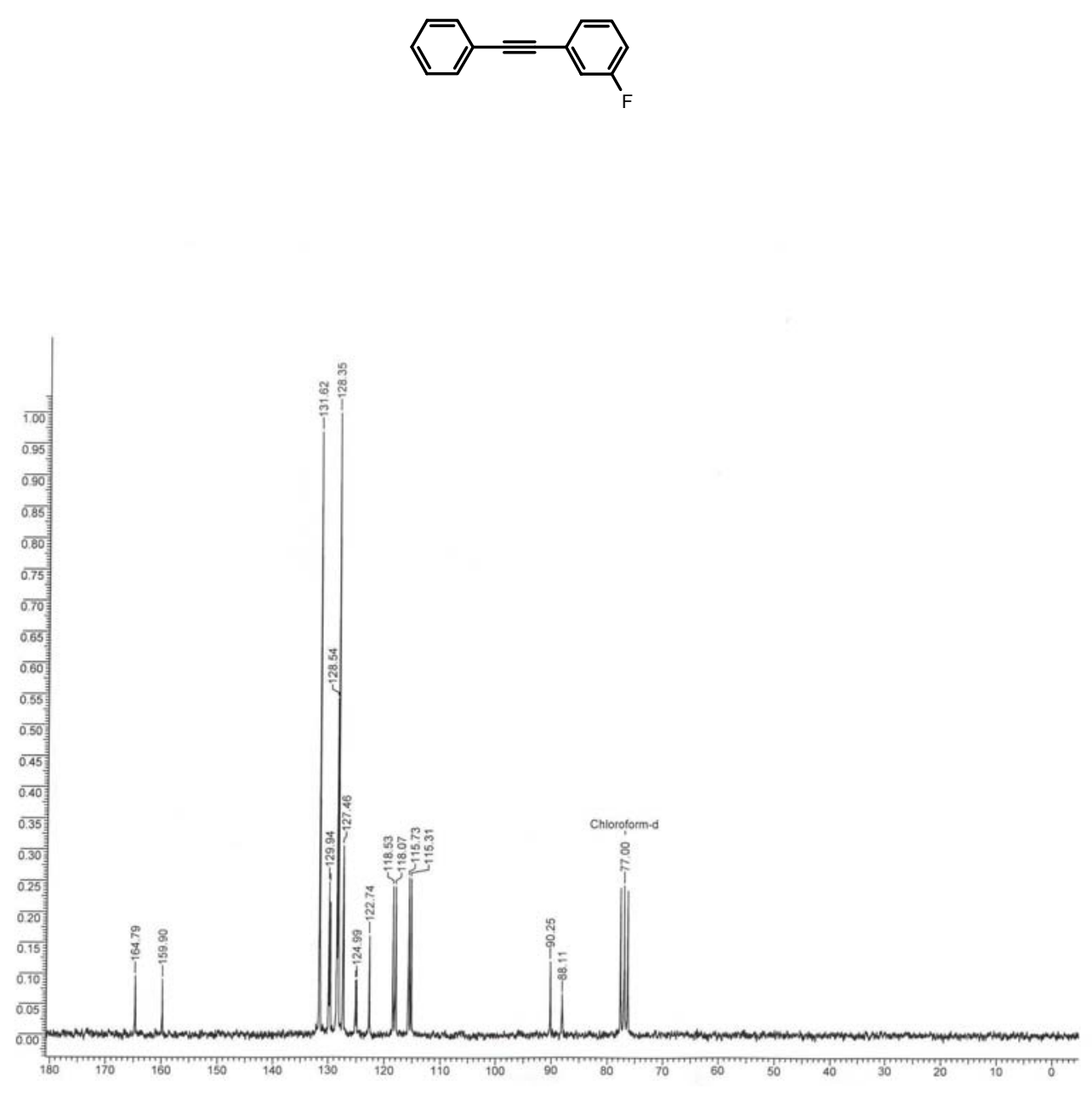


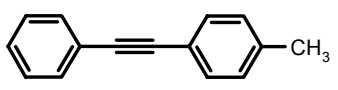

4-Methyl diphenylacetylene

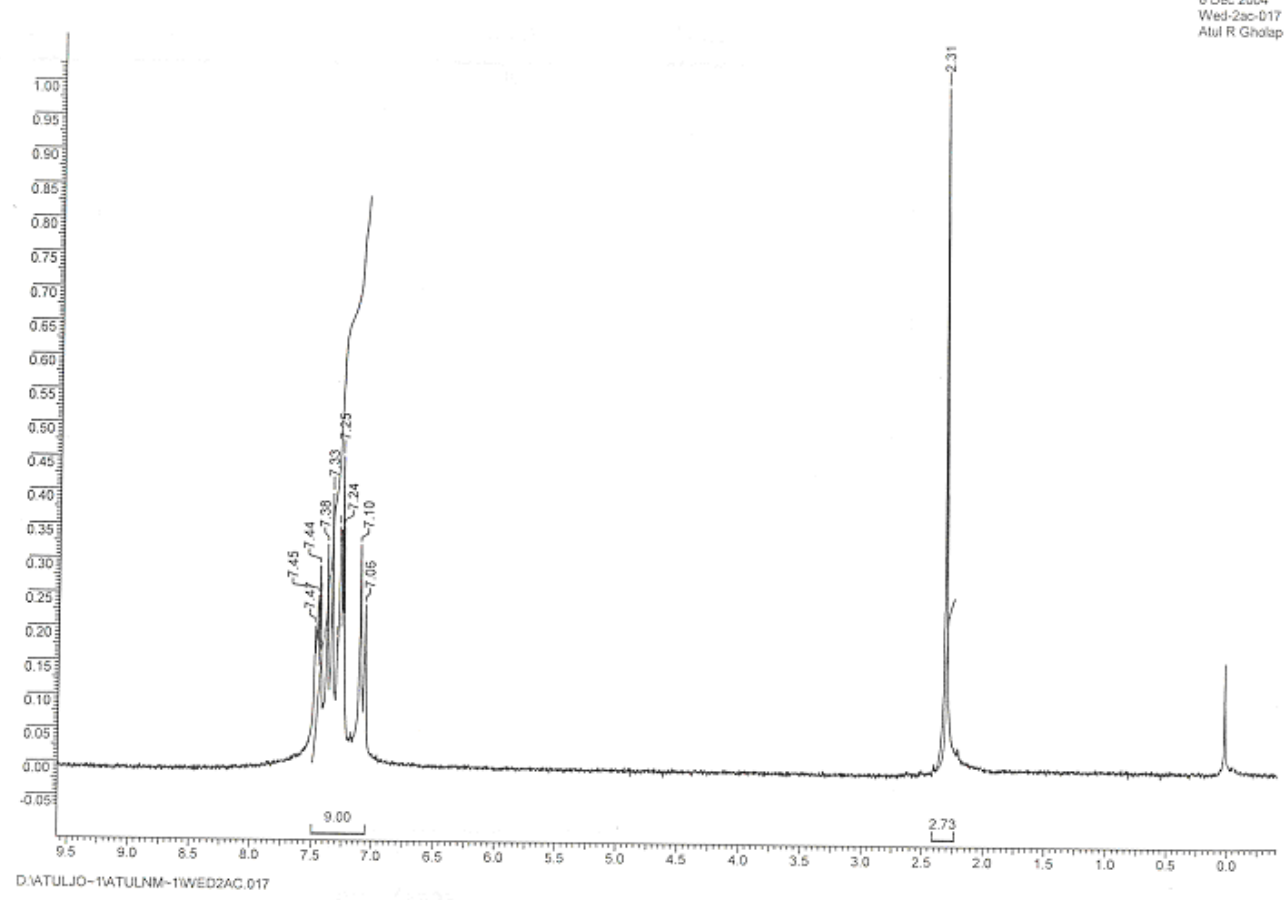

Aromatic region (Entry 3)

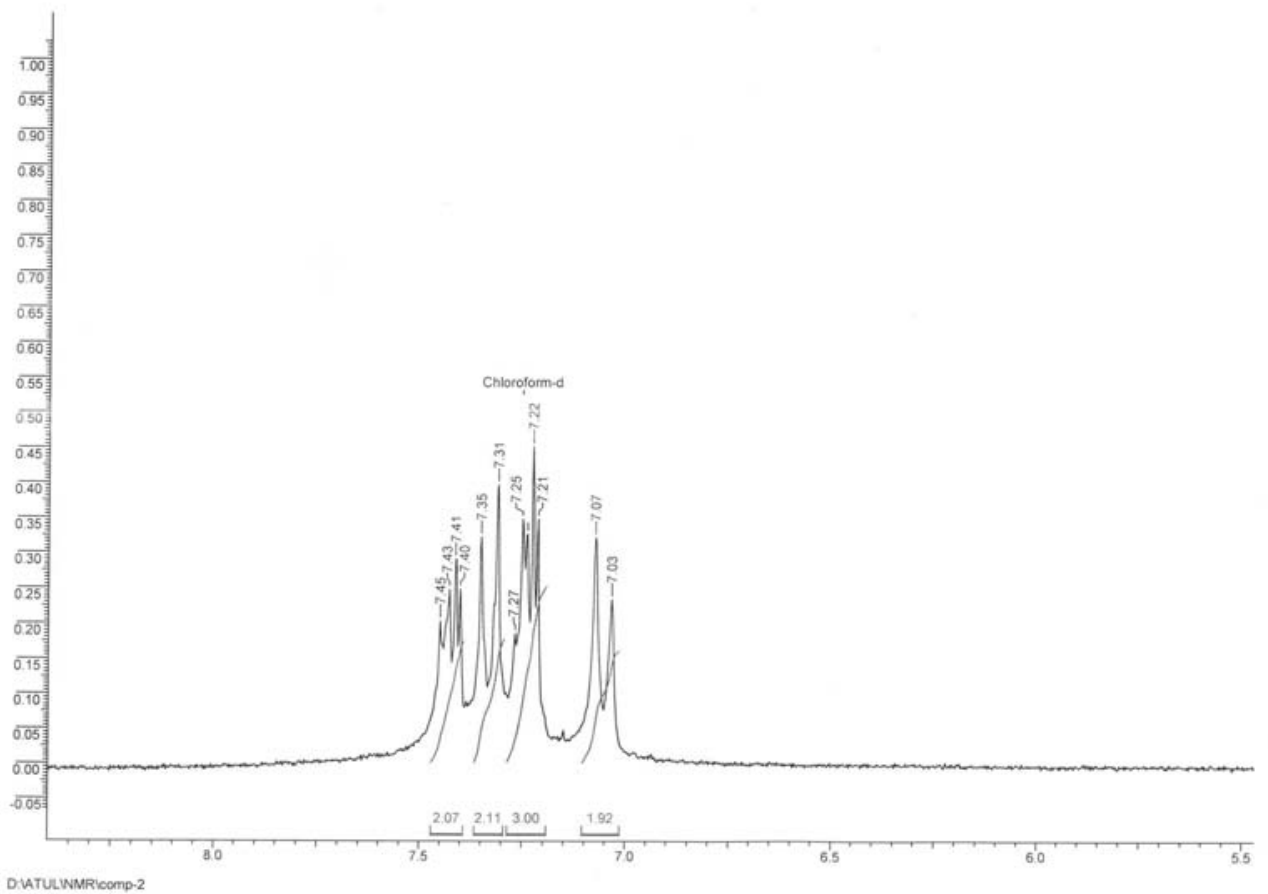




$$
\square=\square-\mathrm{CH}_{3}
$$

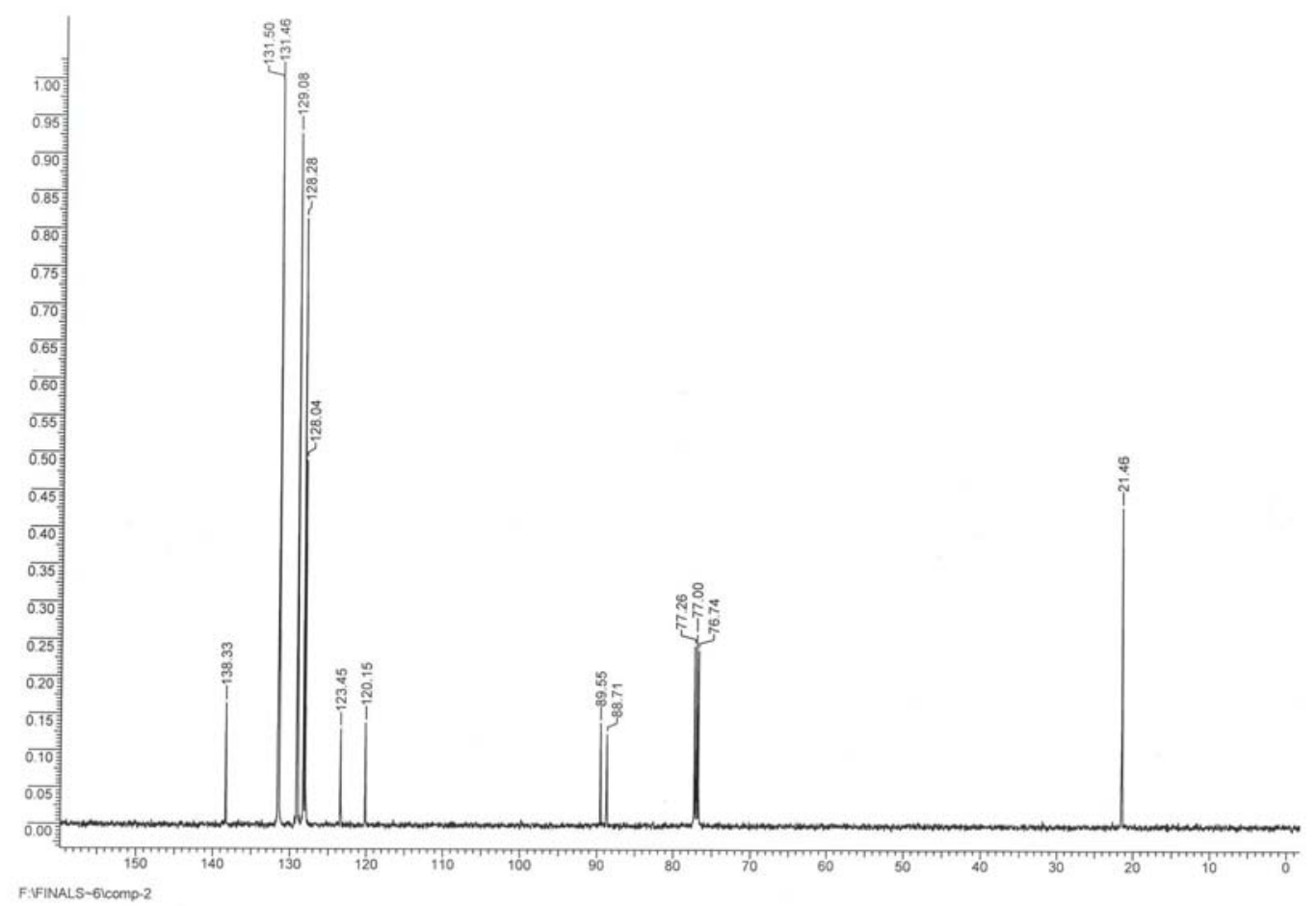



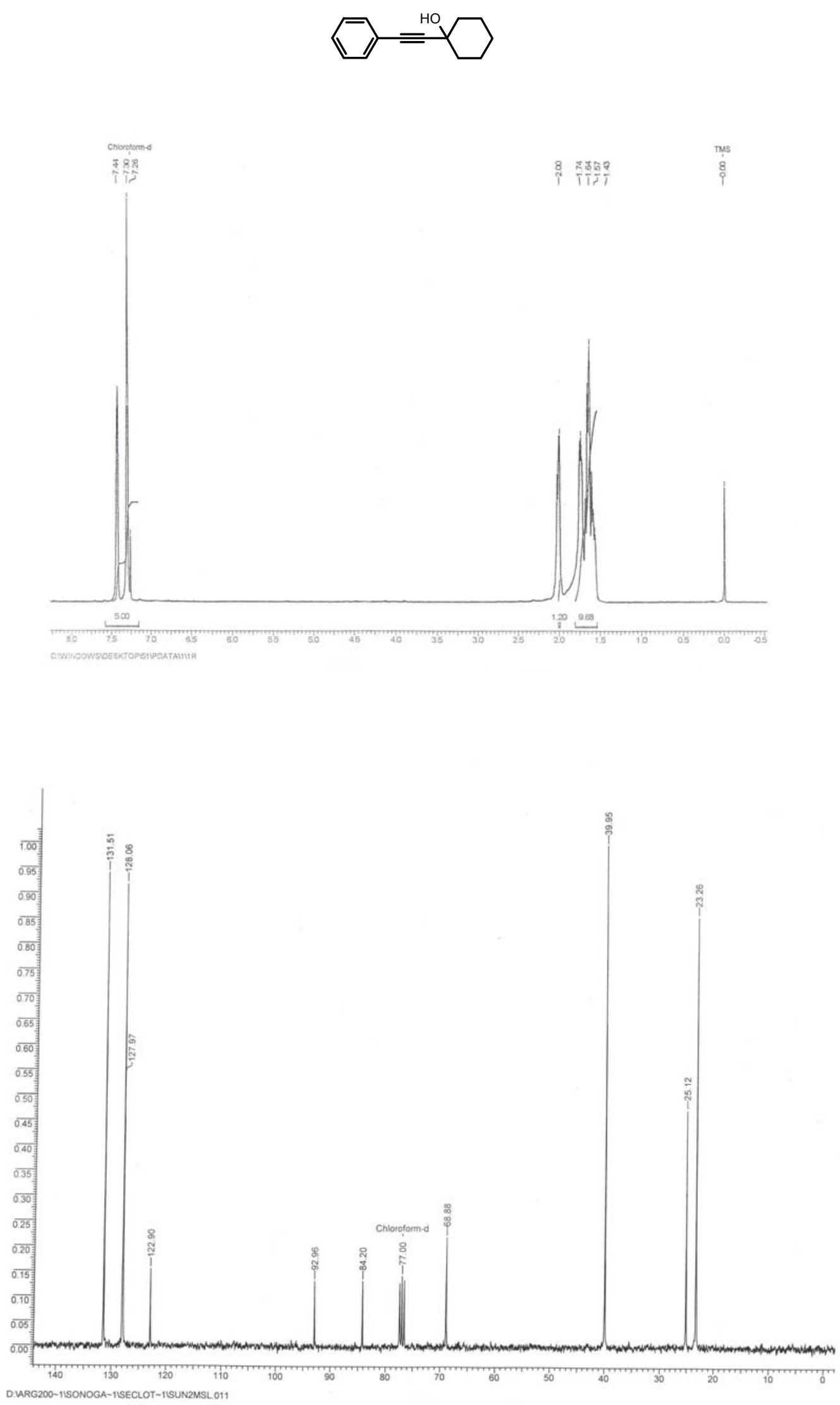

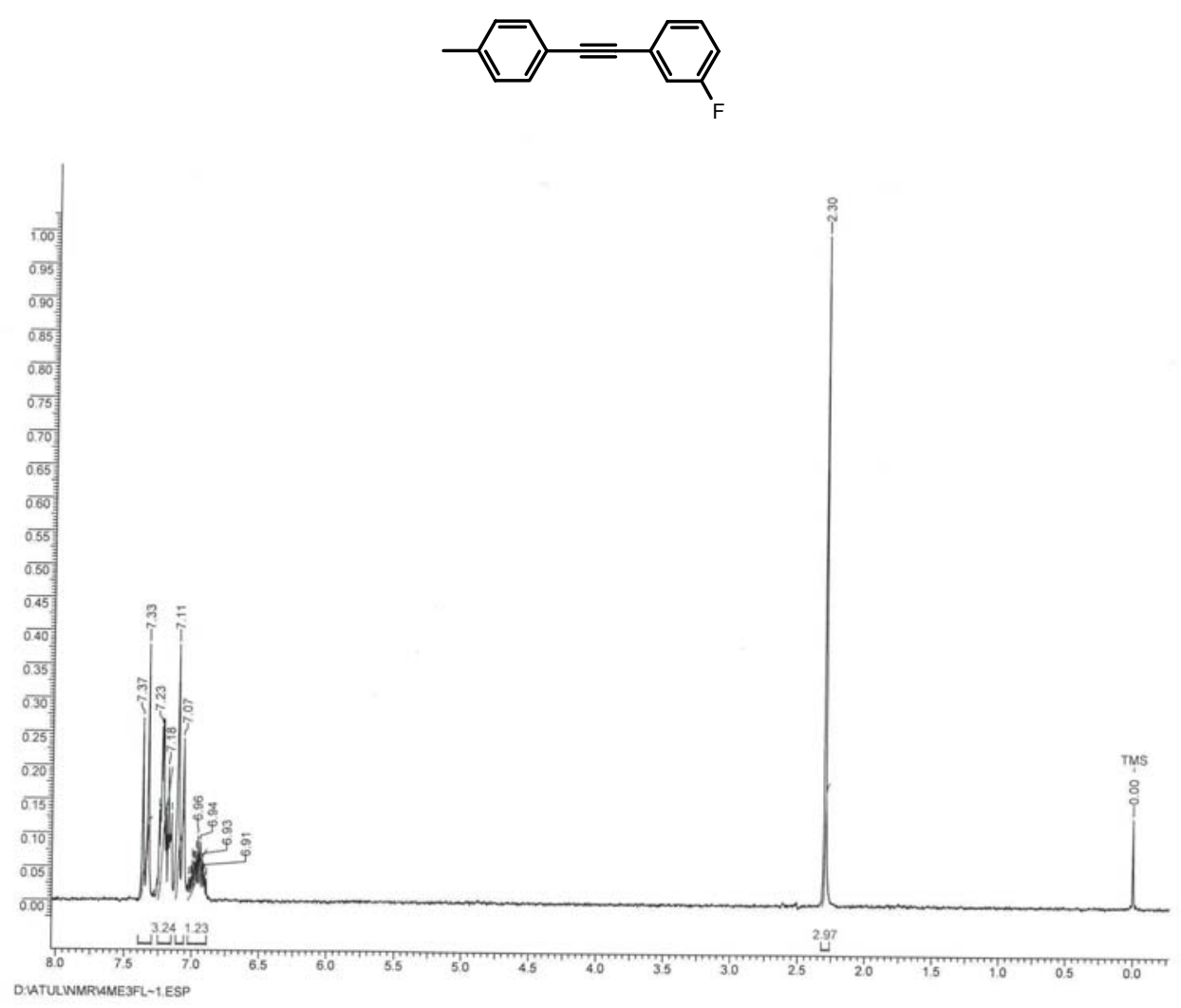

Aromatic region (Entry 6)

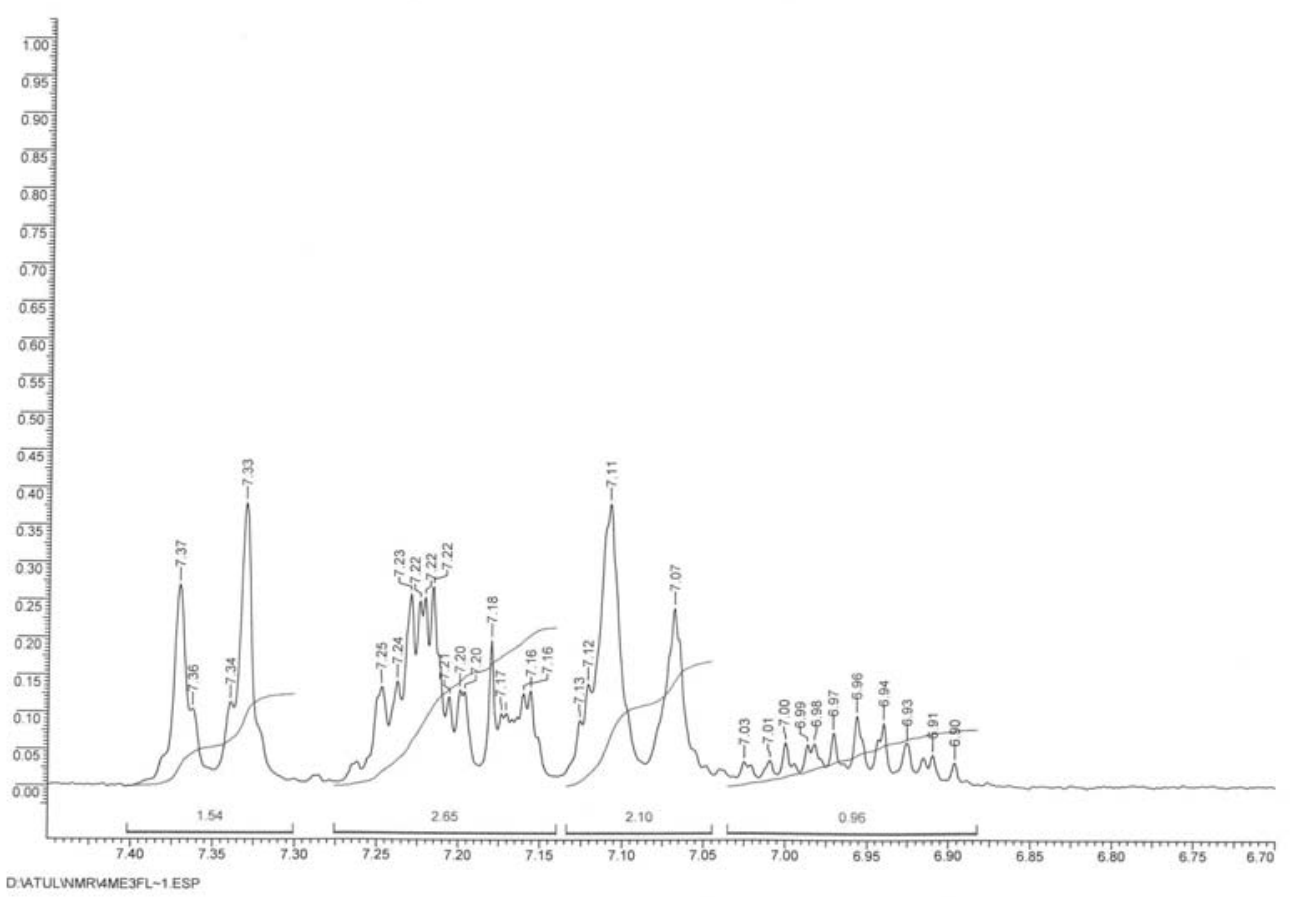




\section{Entry 6}

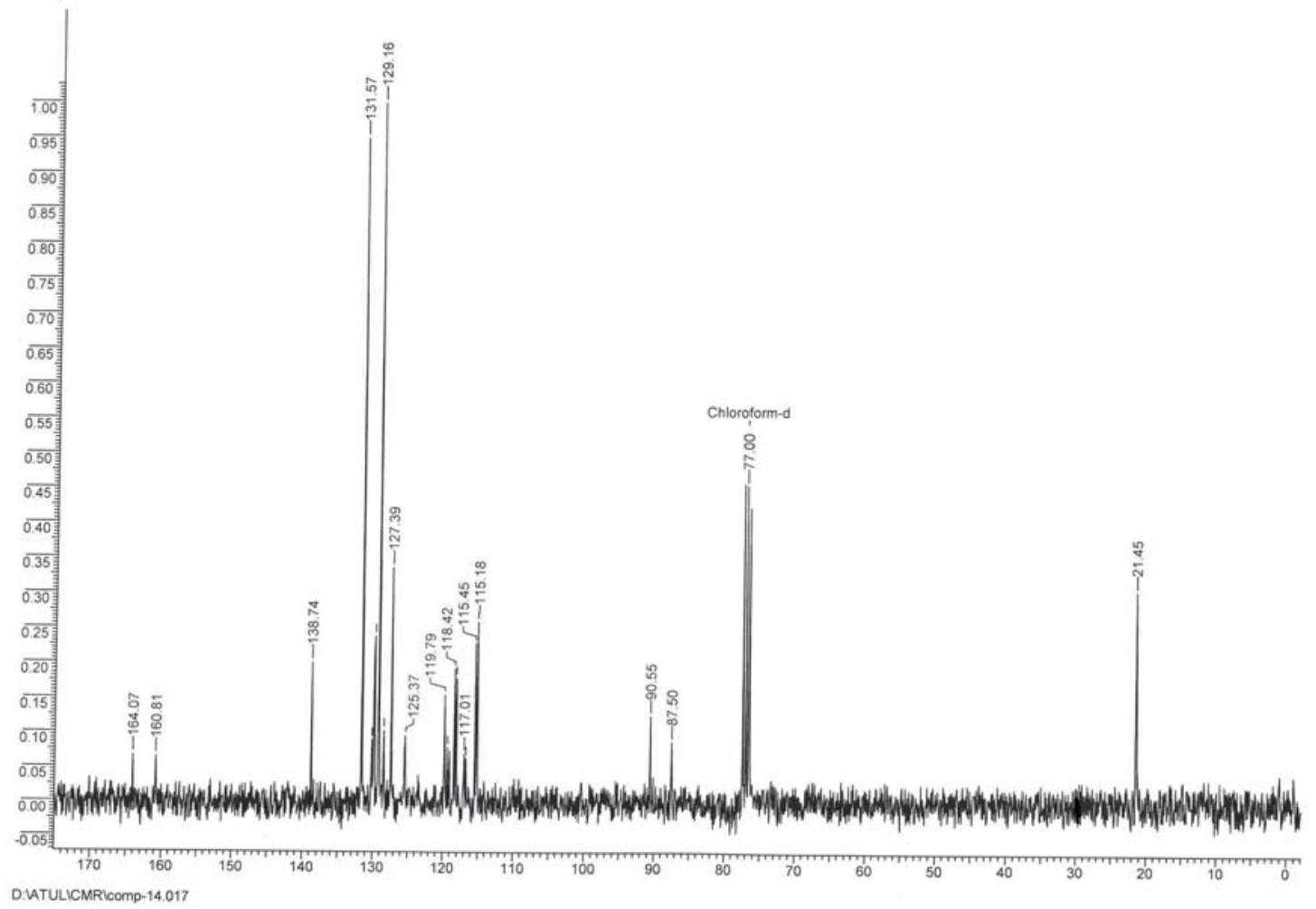



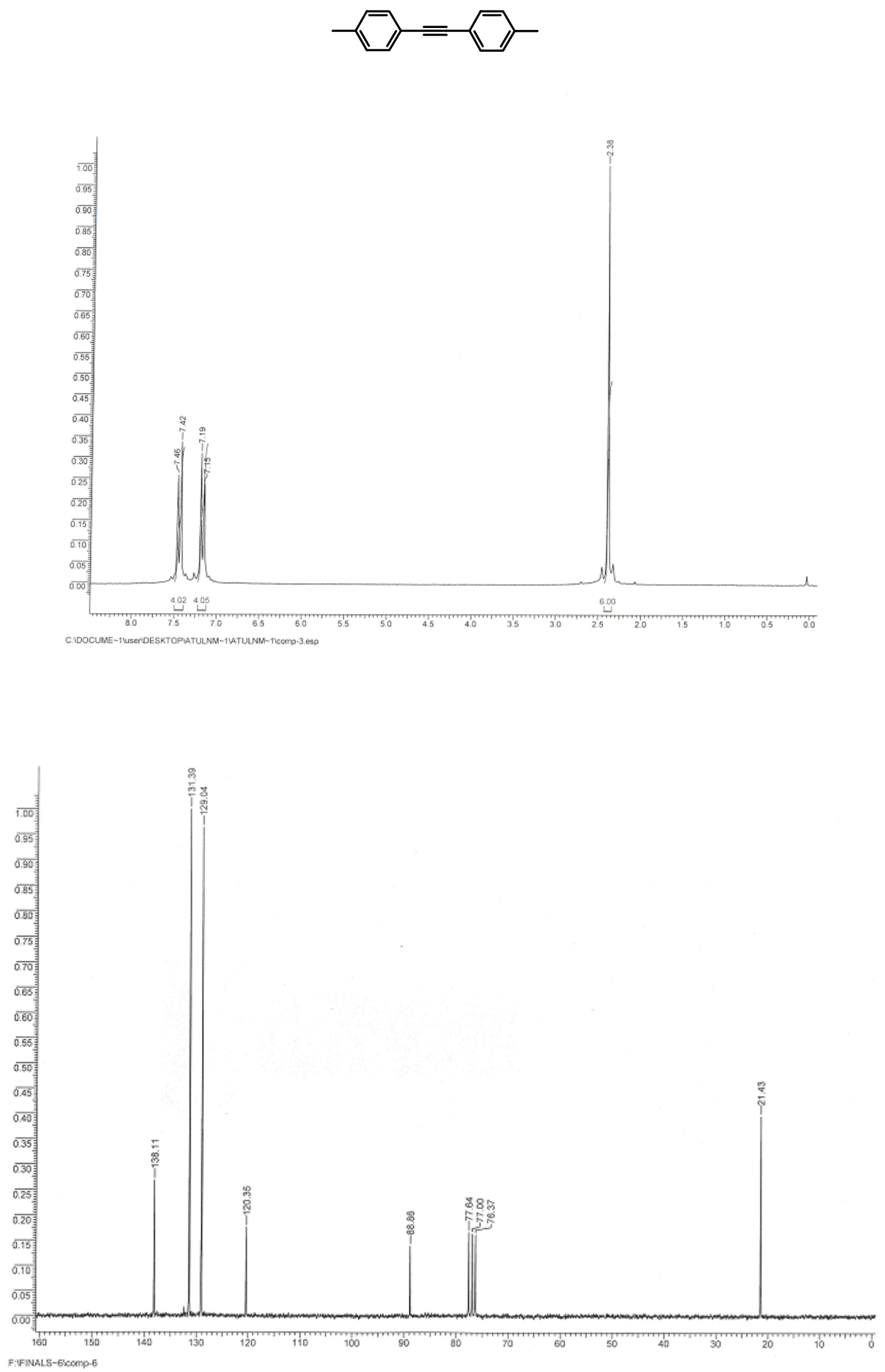

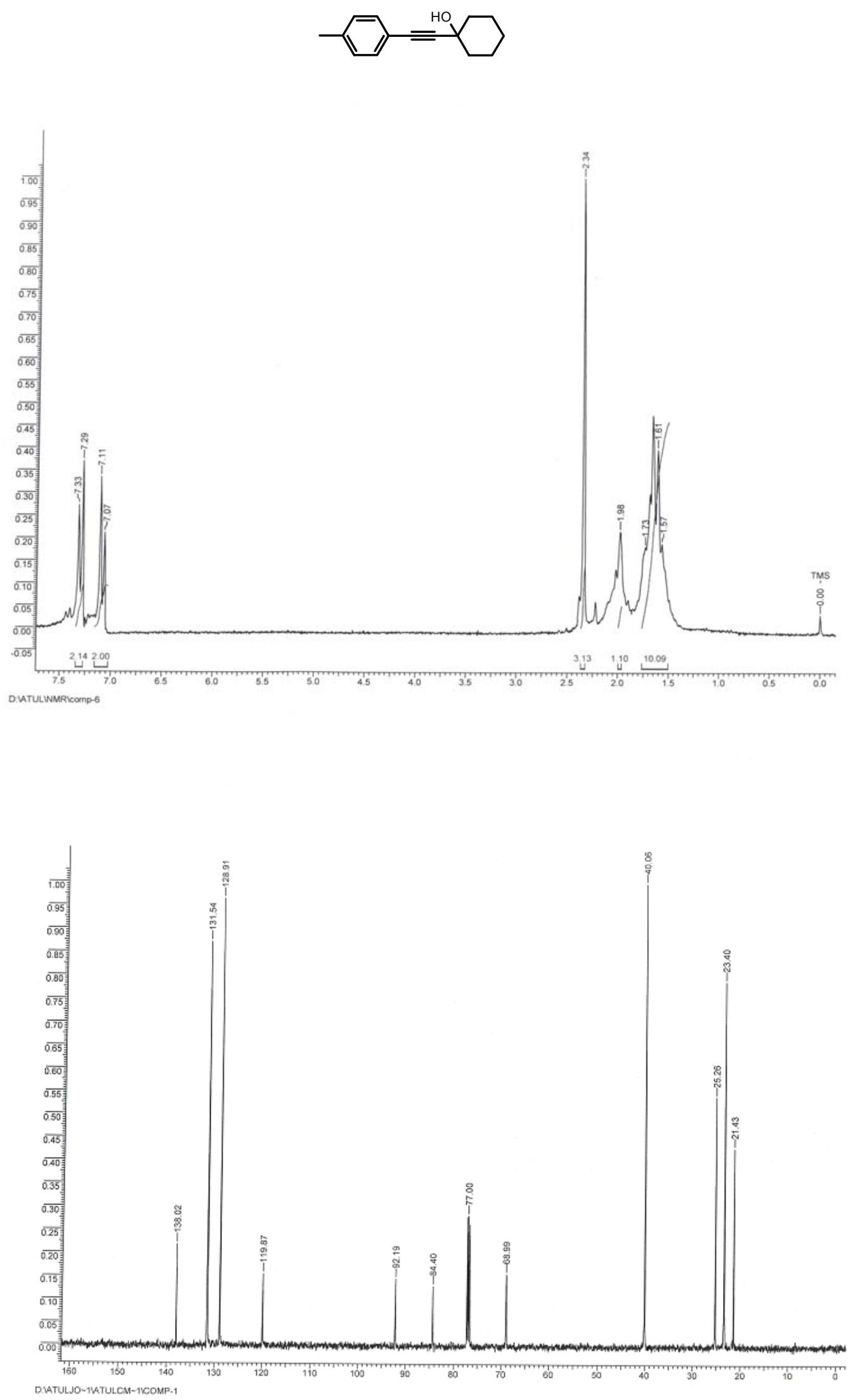

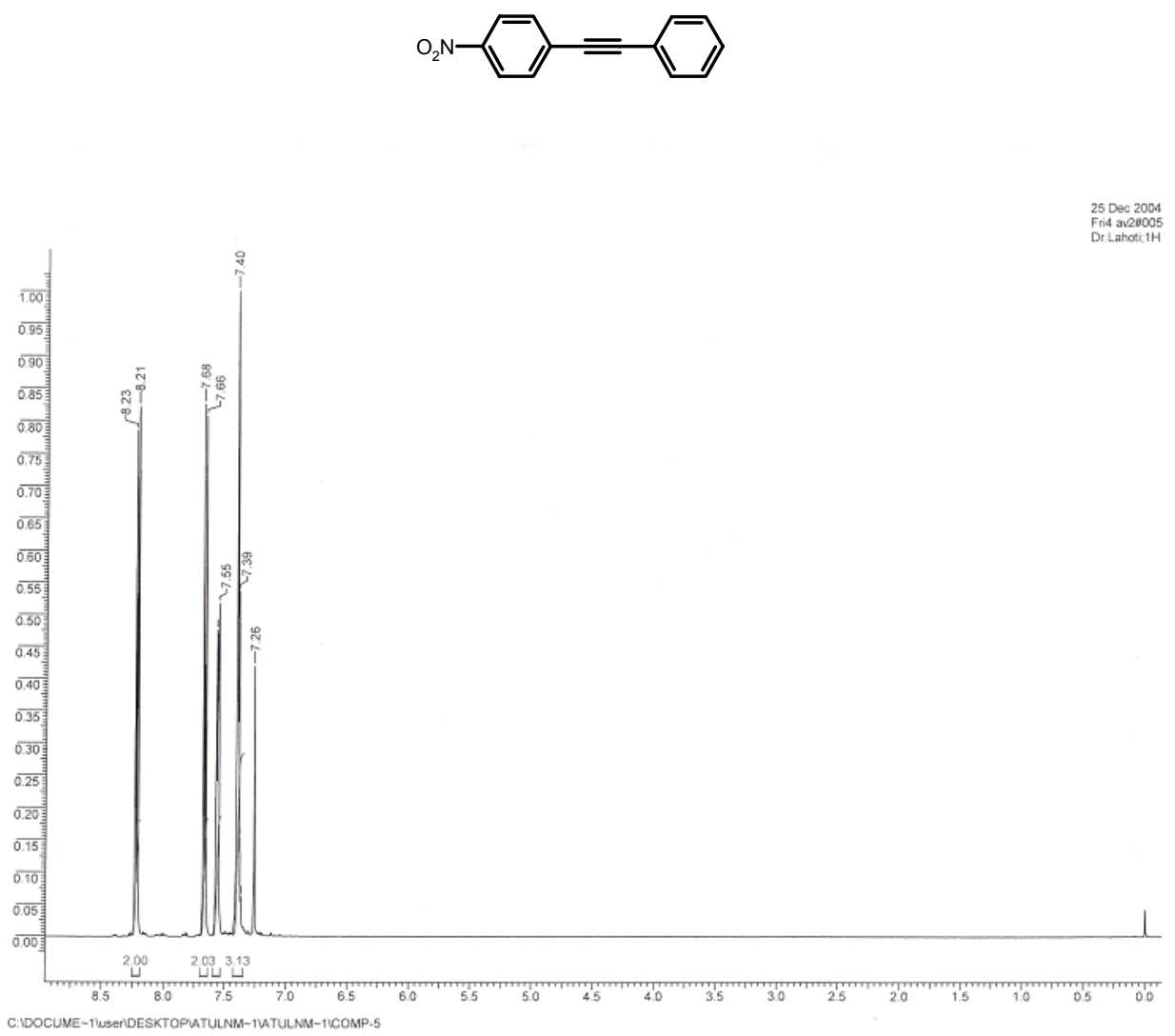

Aromatic region (Entry 9)

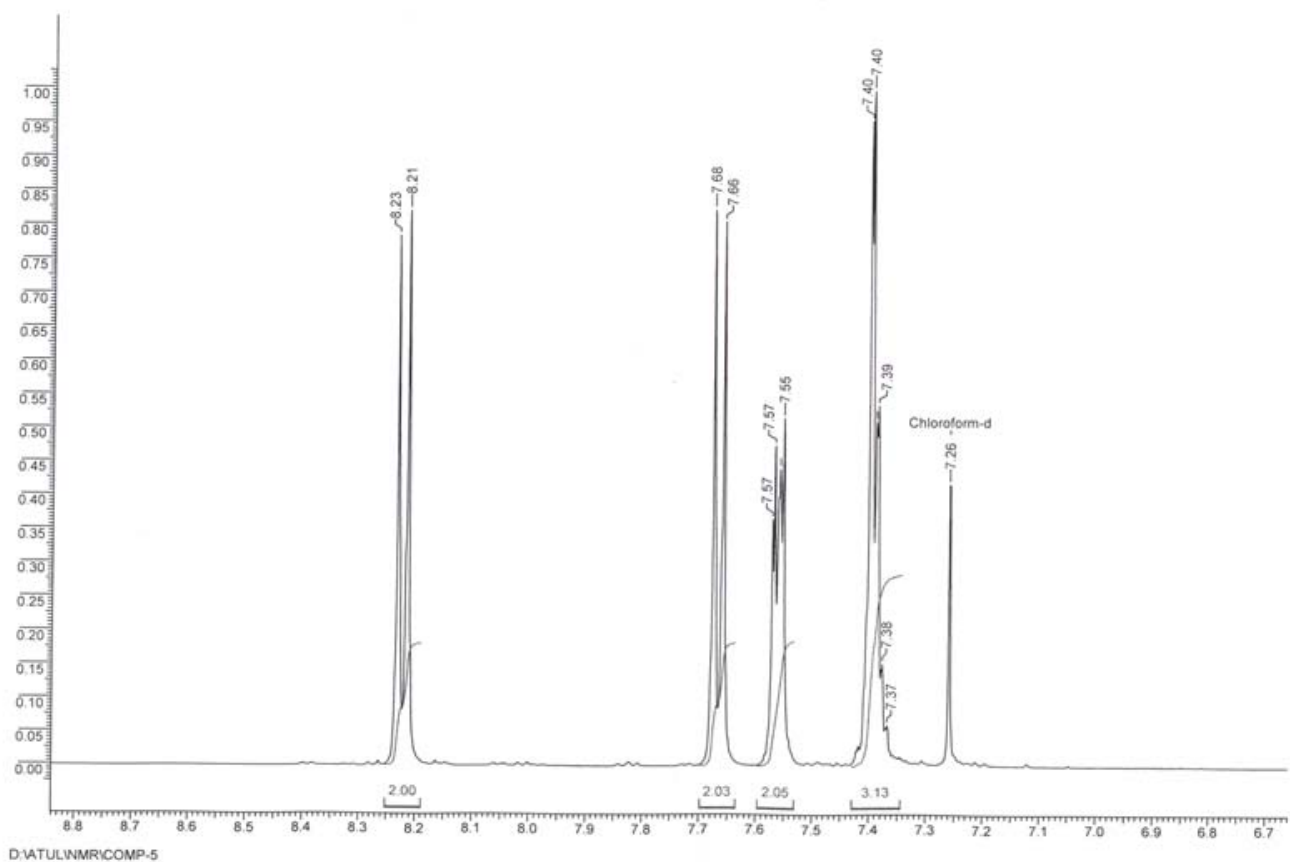



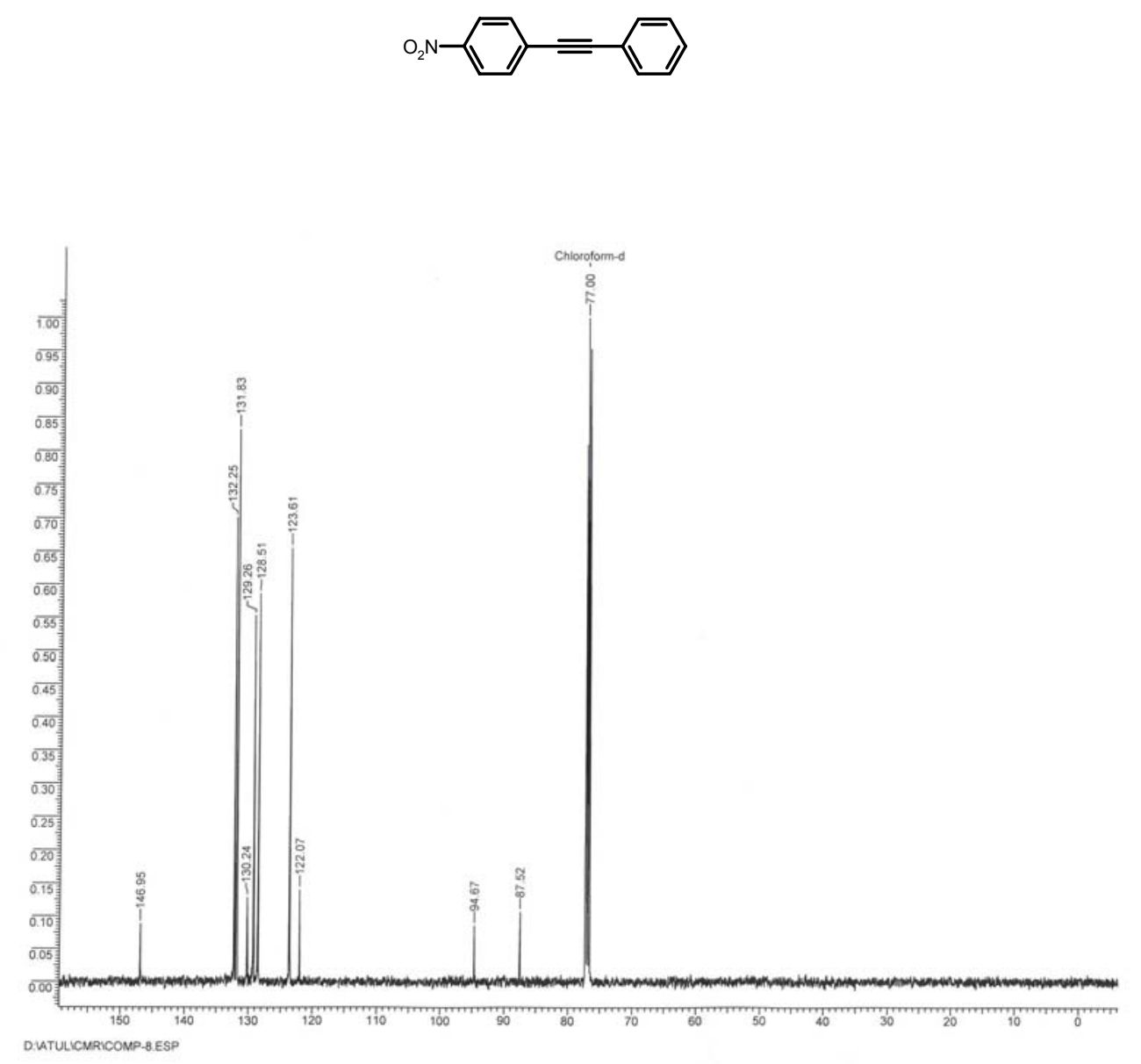
$\longrightarrow=$
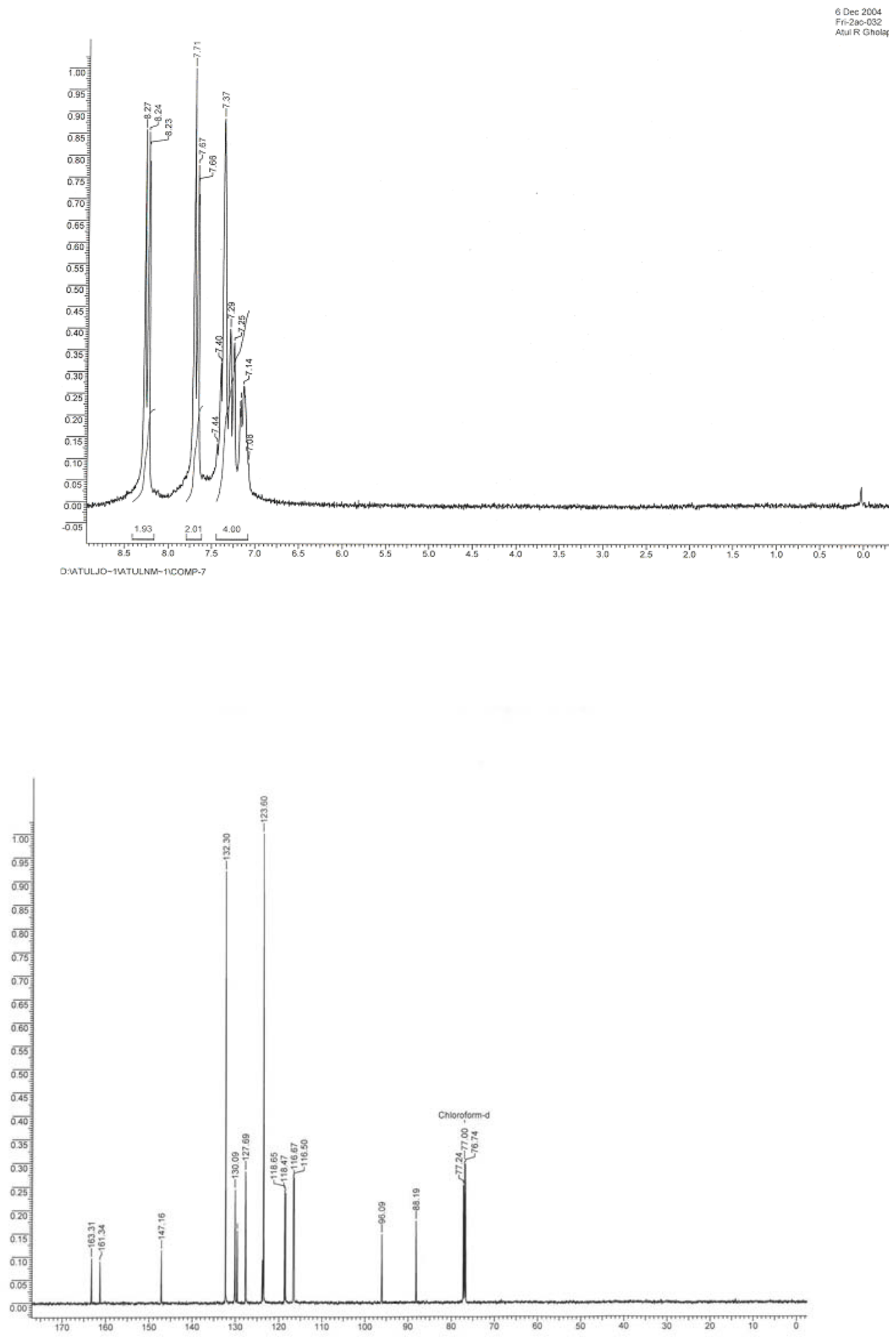
$\square=$
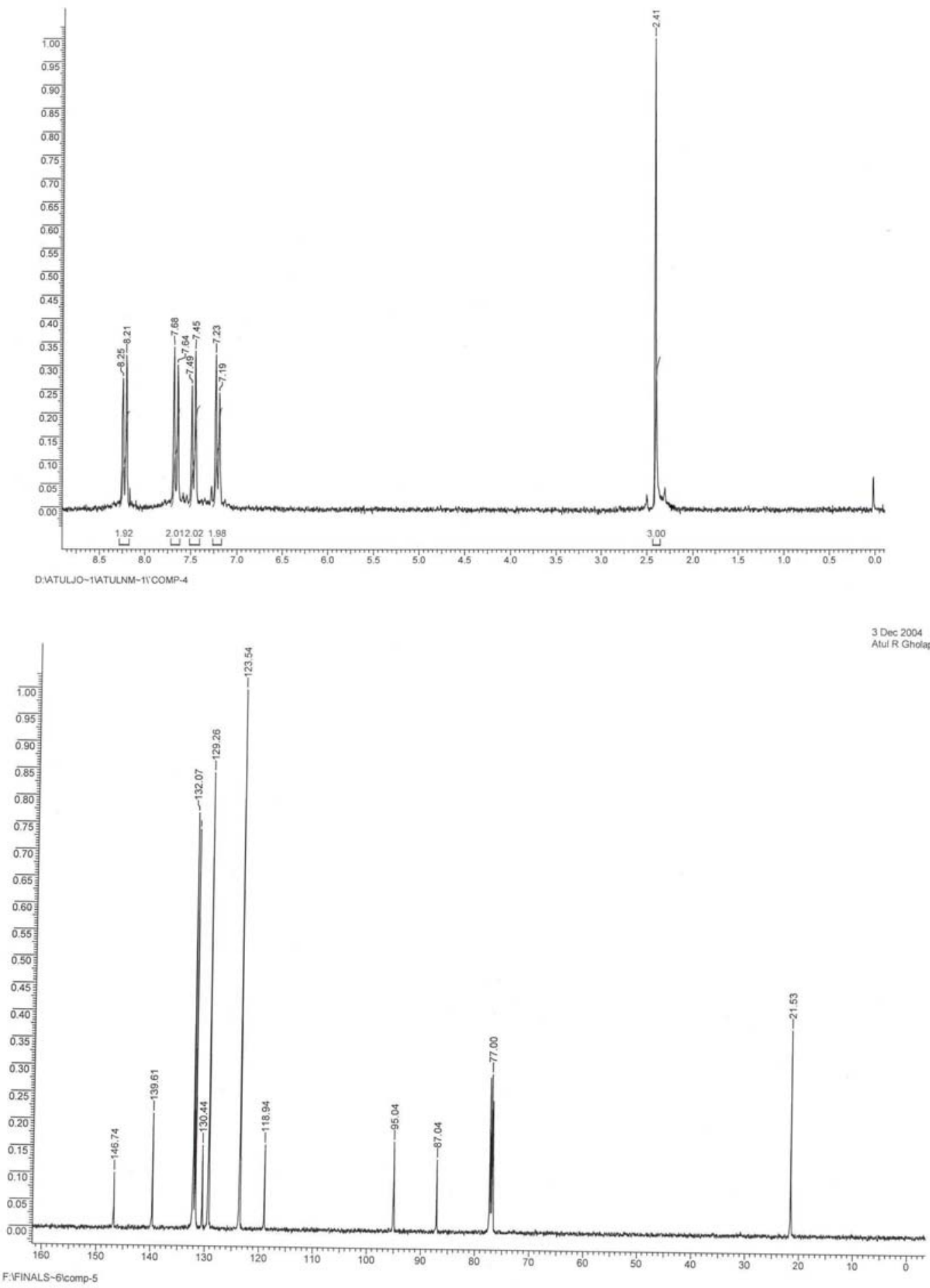

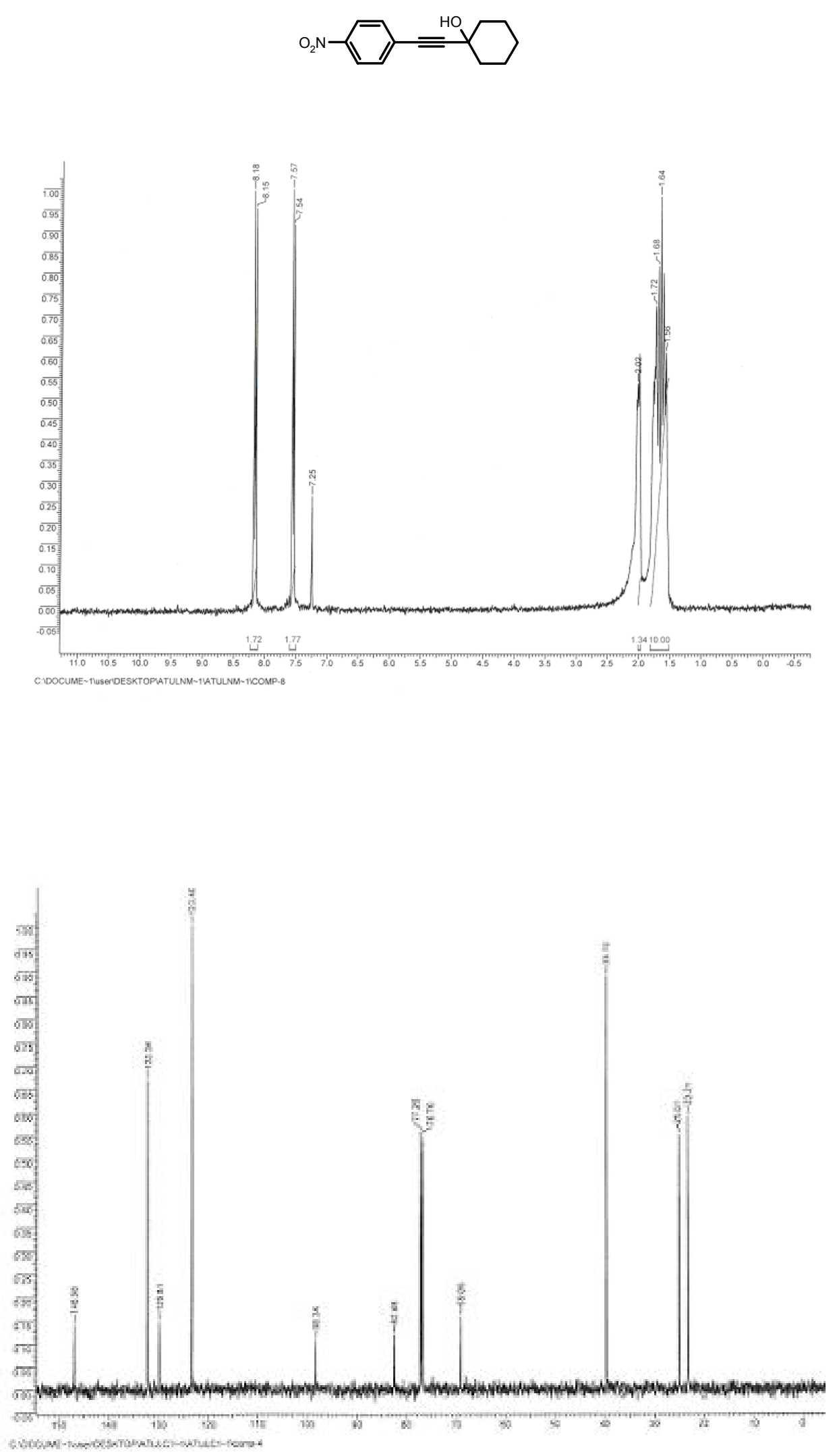


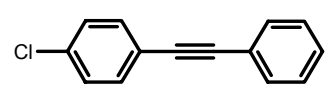

4-Chloro-diphenylacetylene

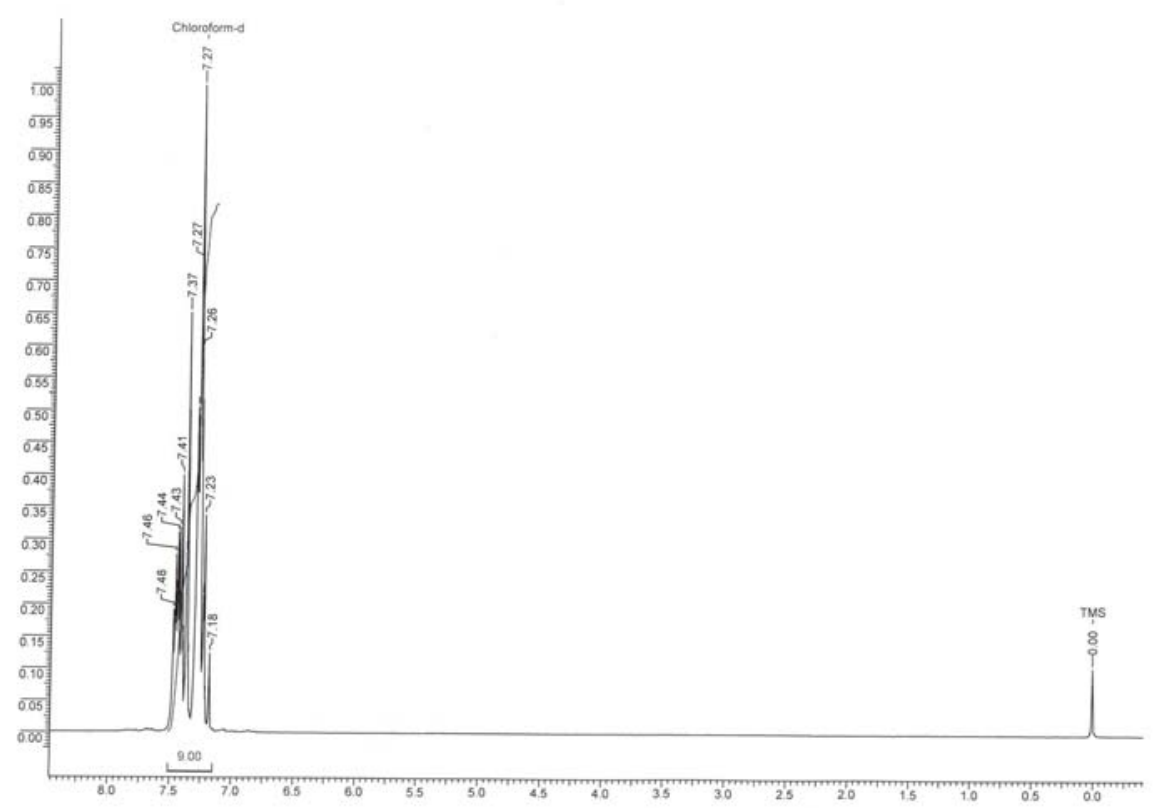

Aromatic region (Entry 13)

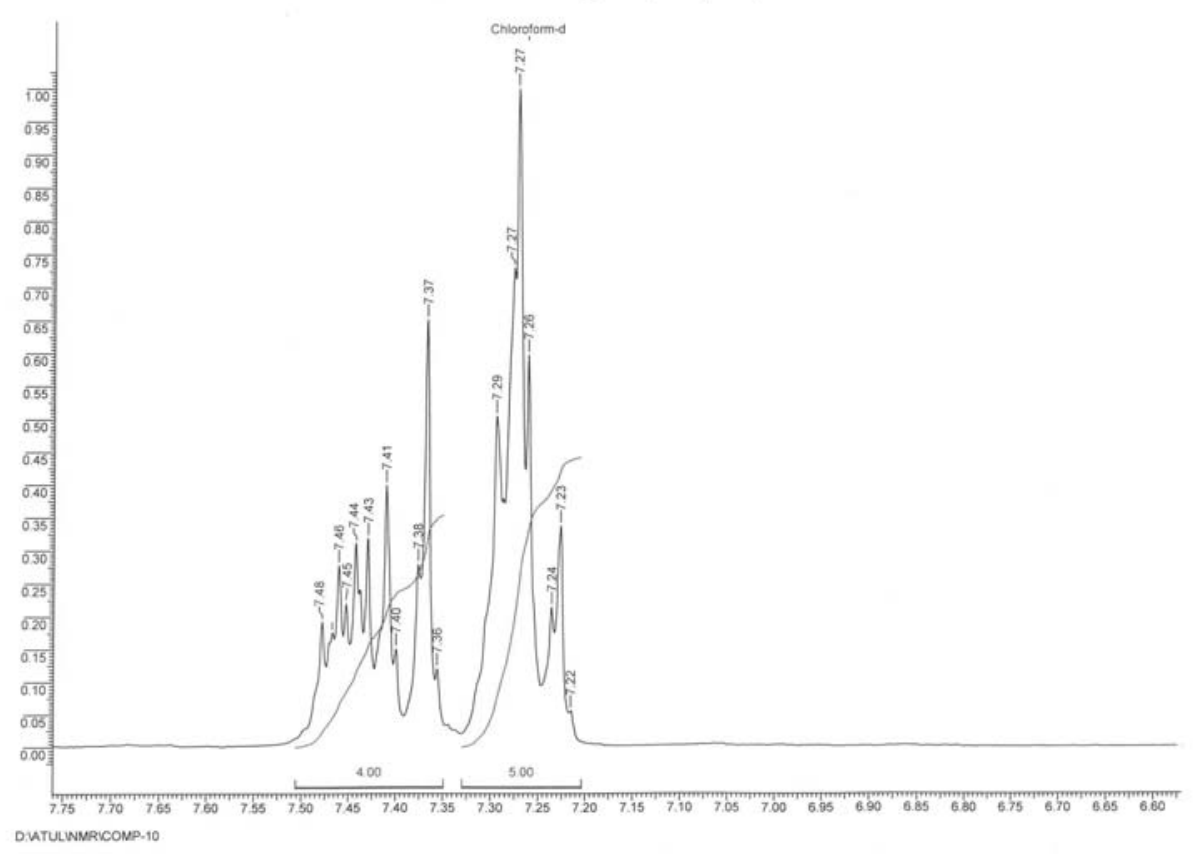



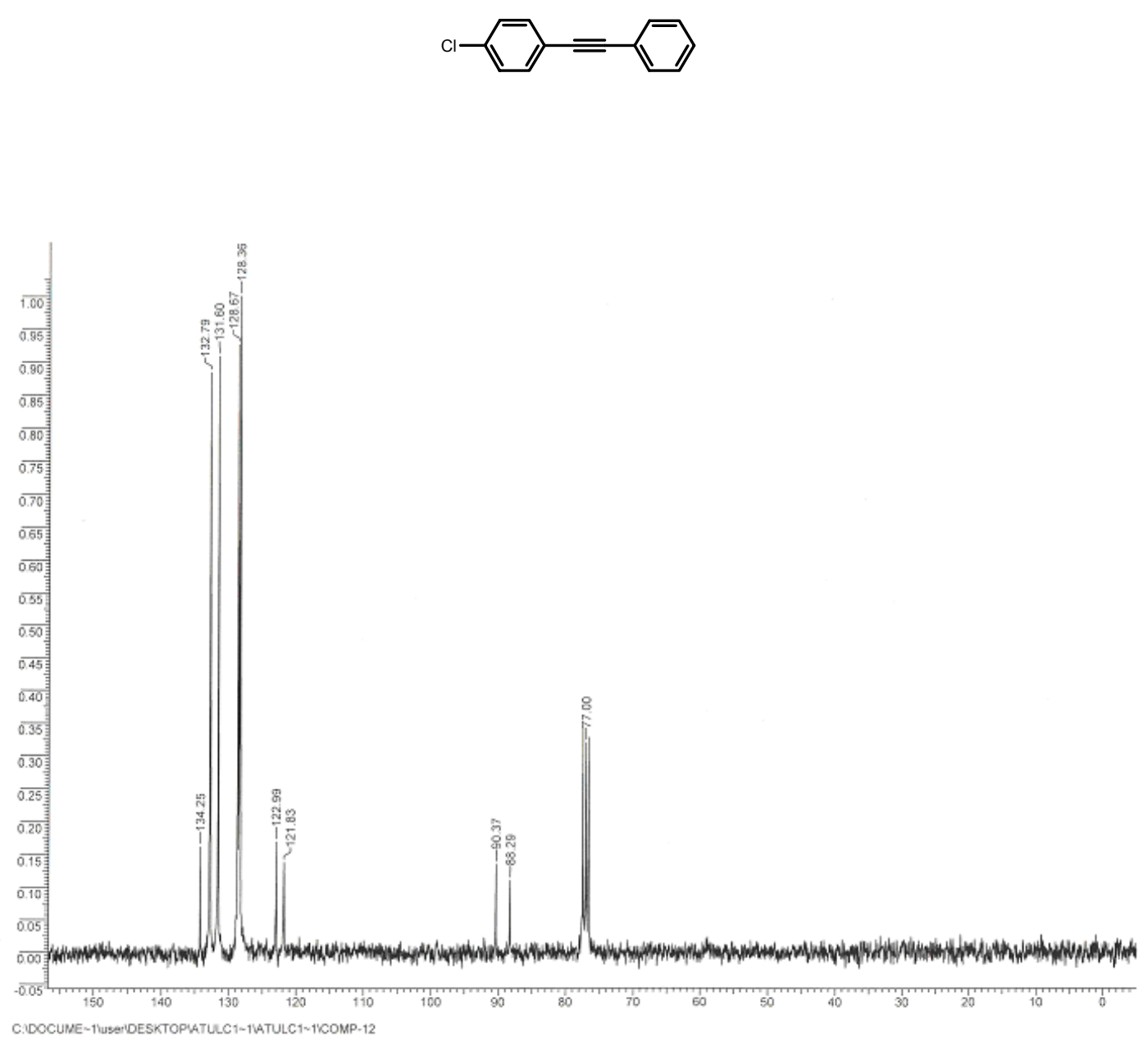

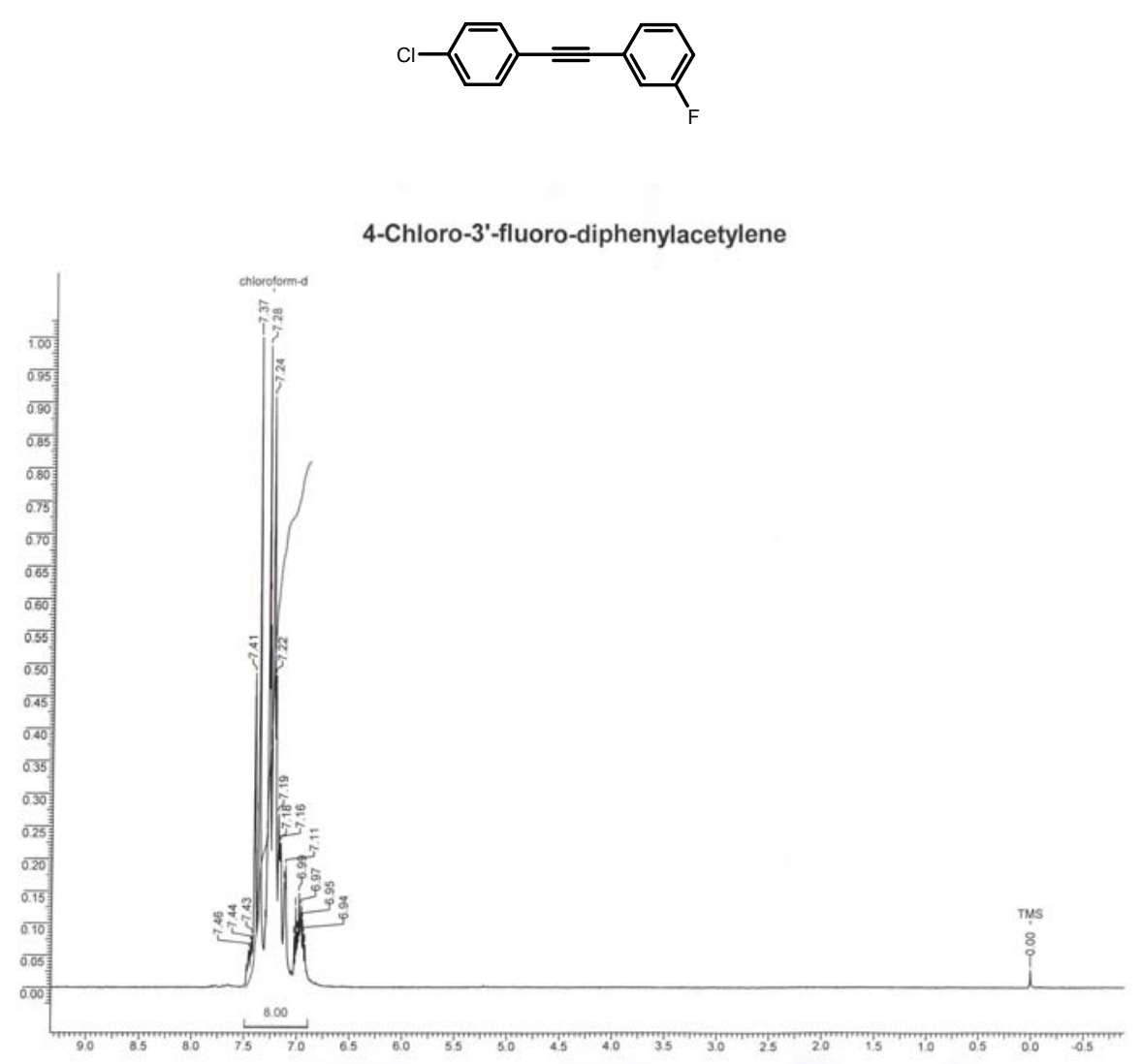

Aromatic region (Entry 14)

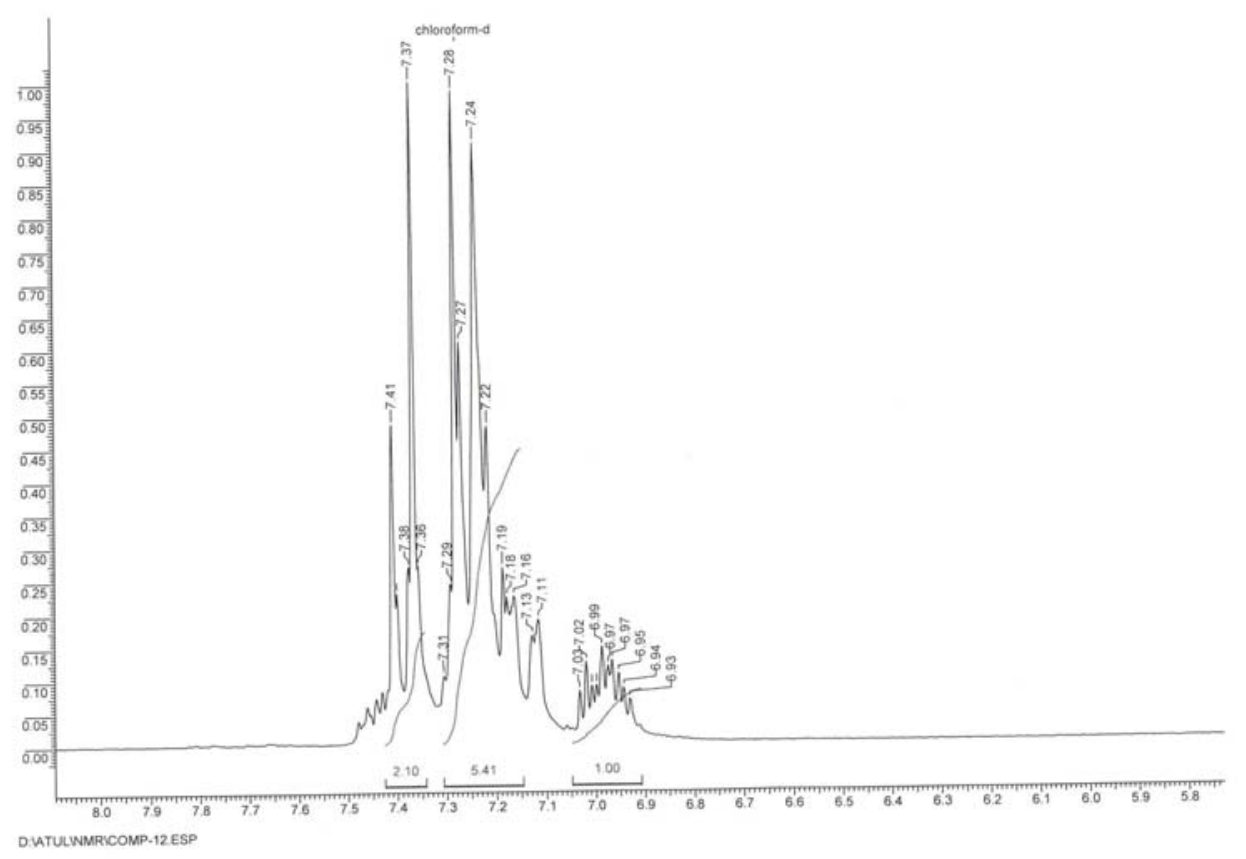



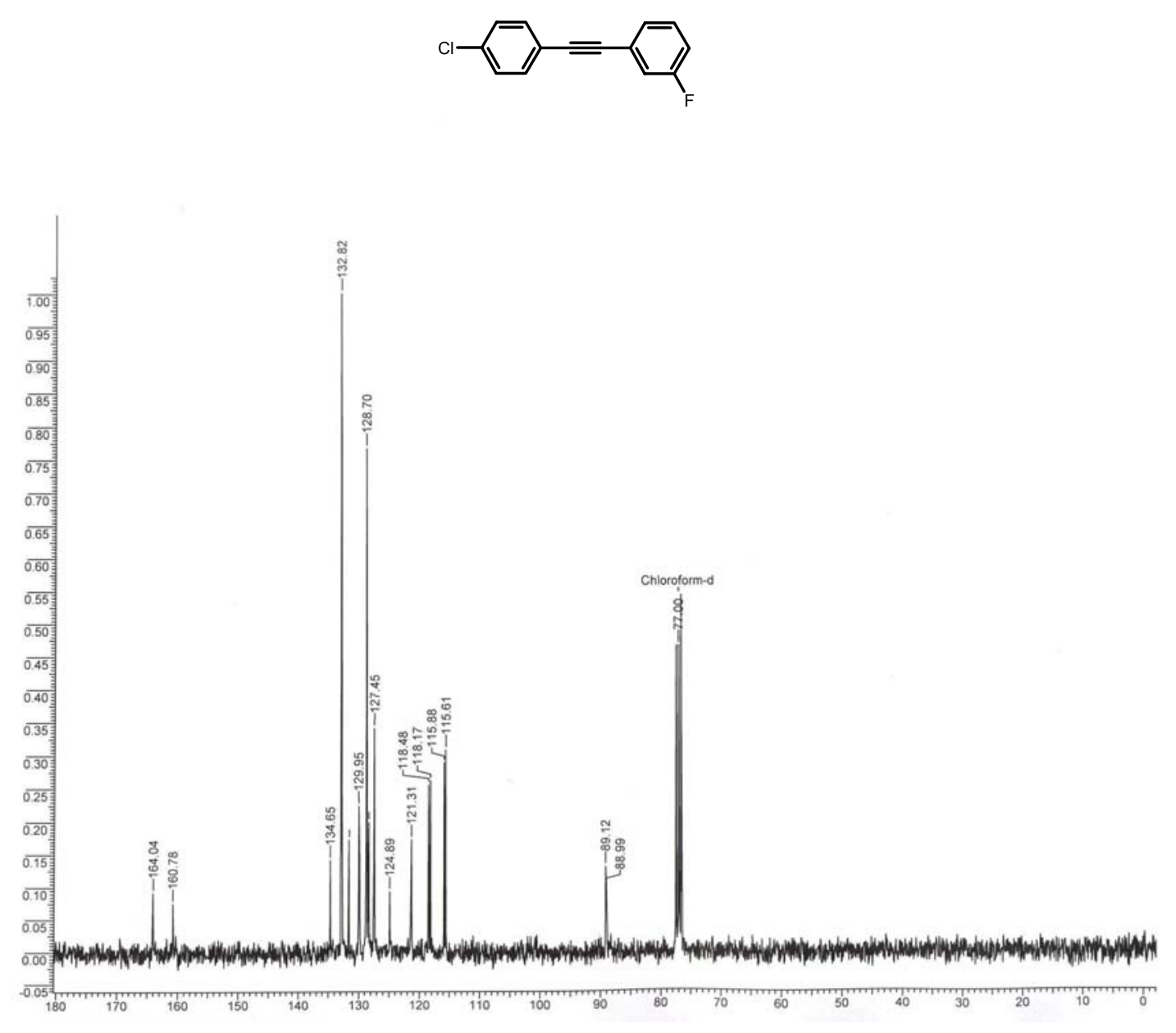

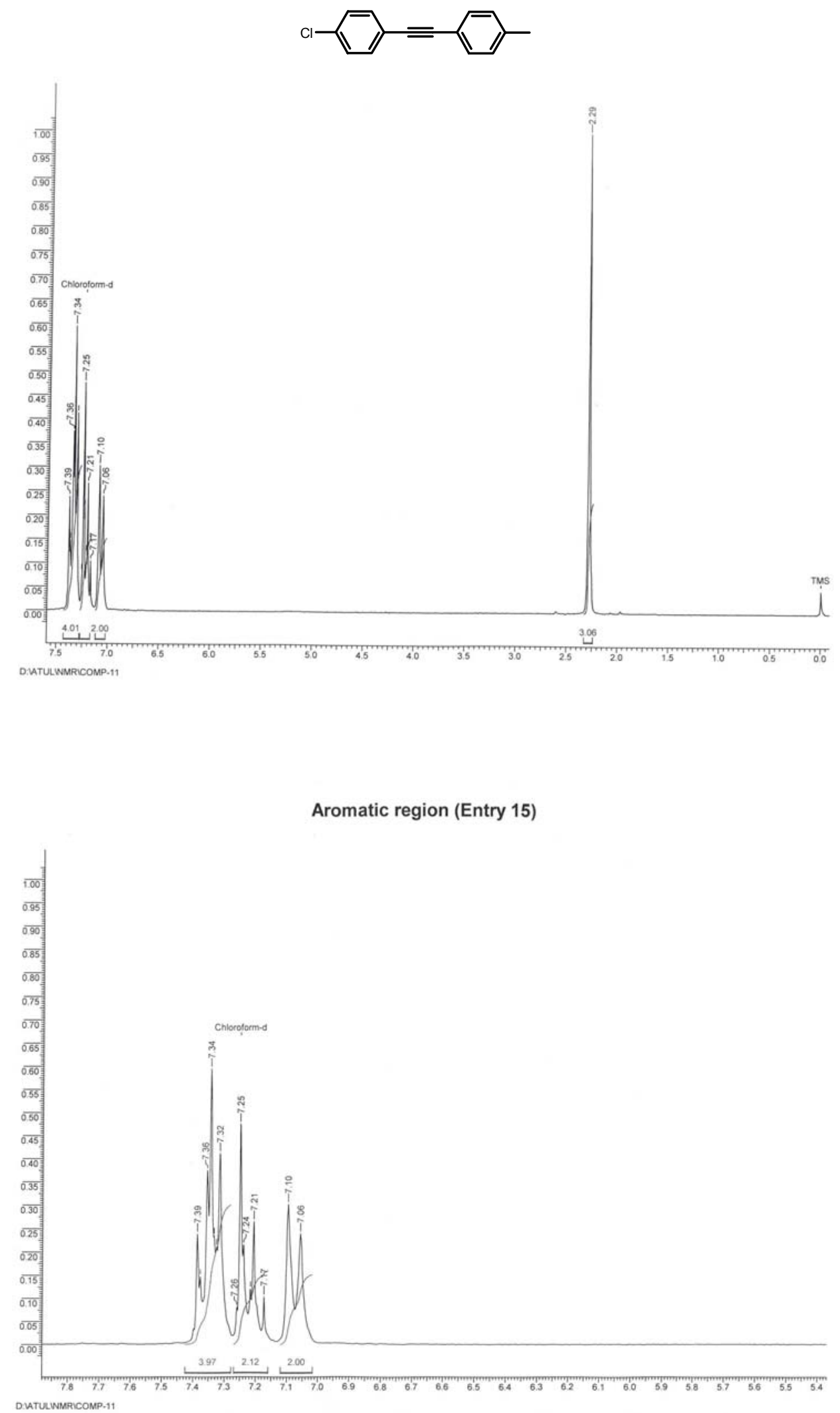

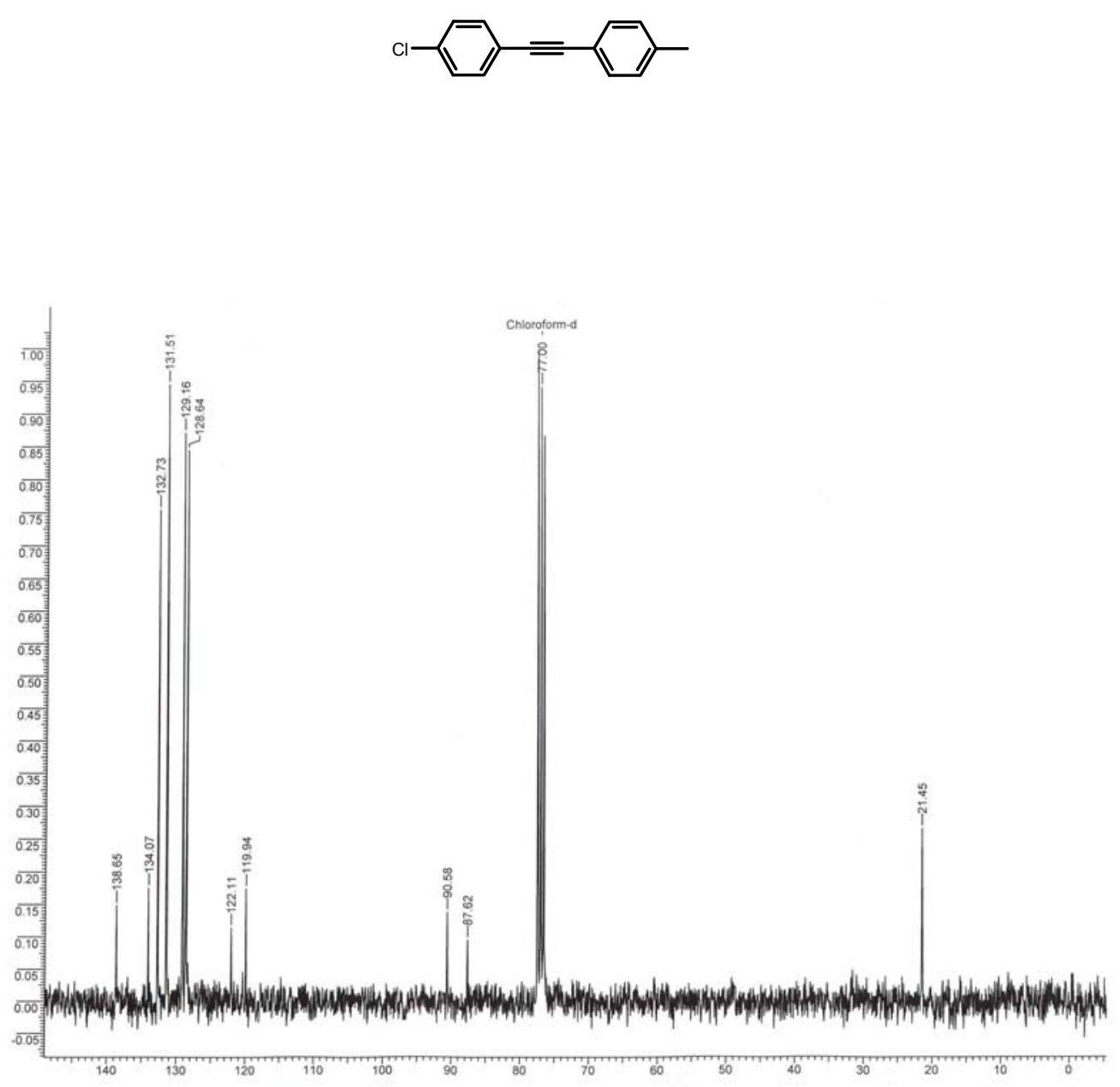

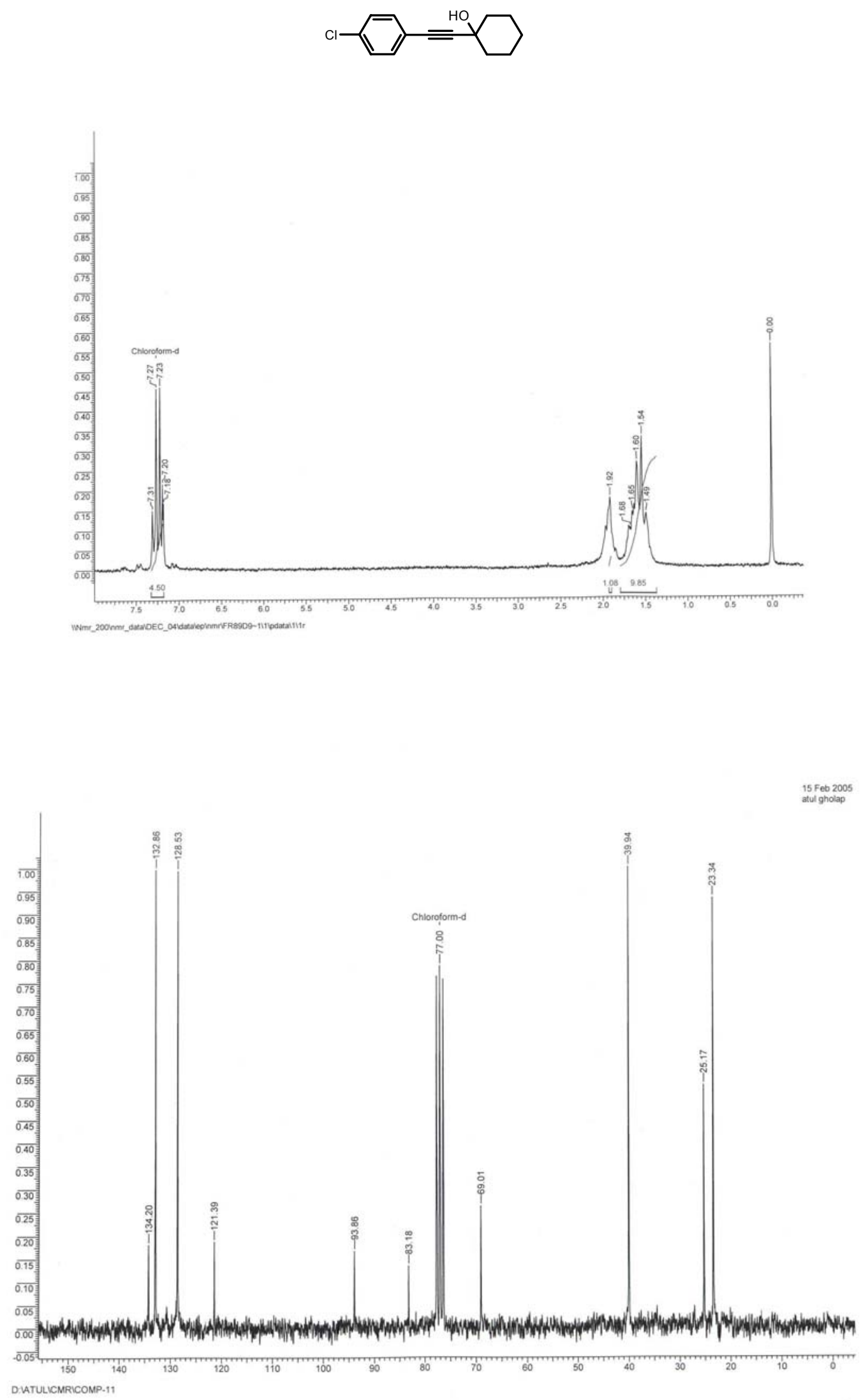

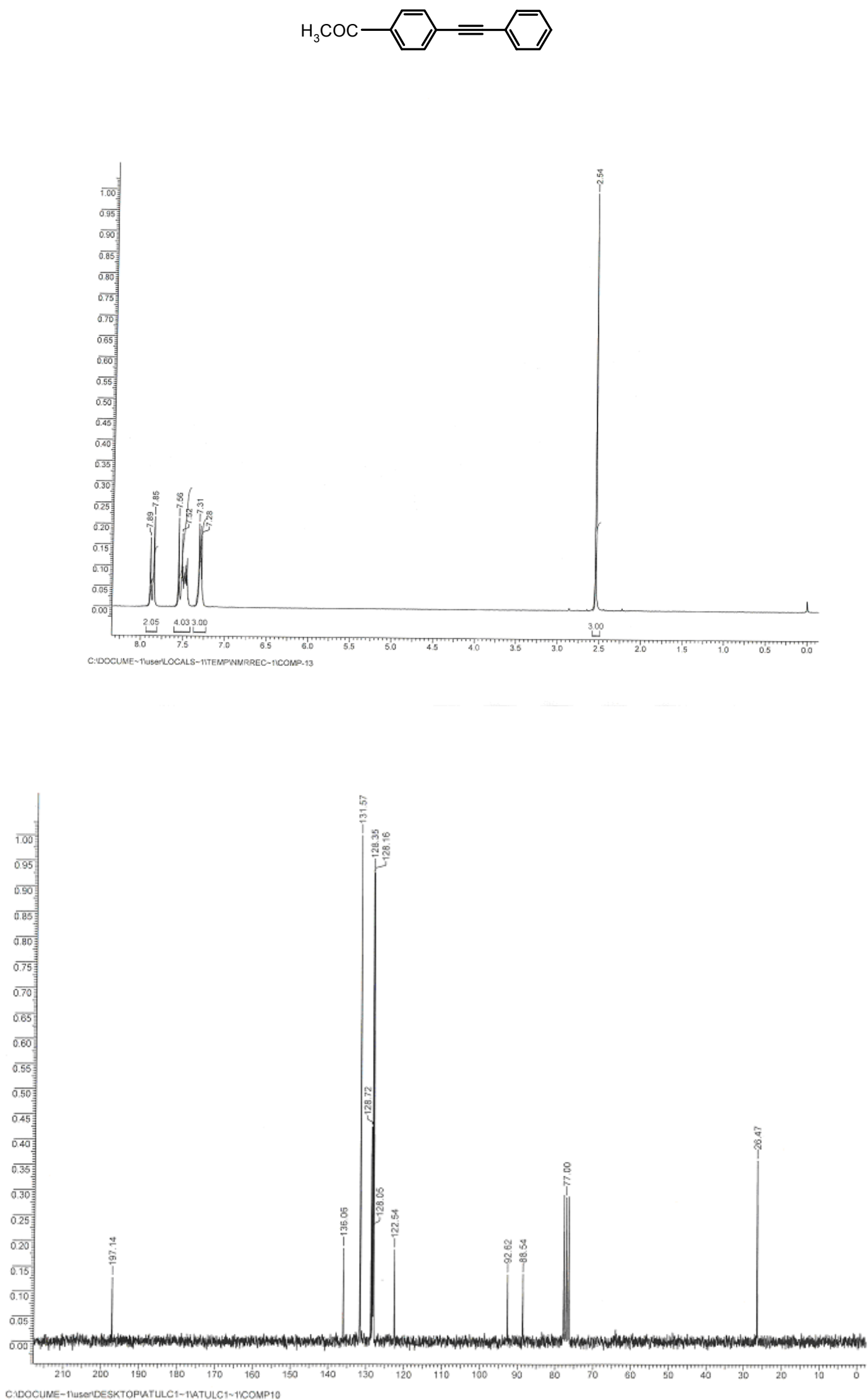

S33 

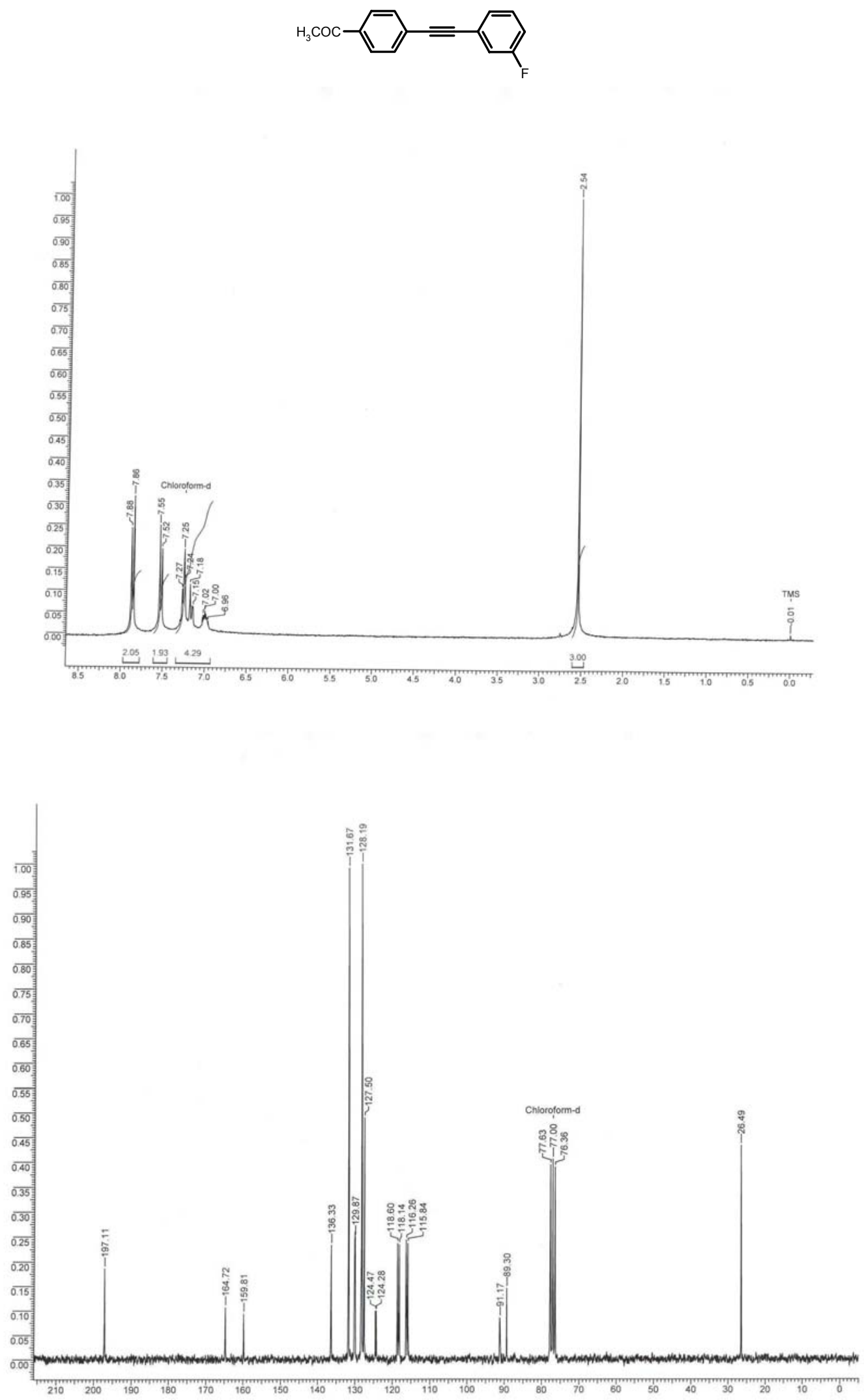

S34 

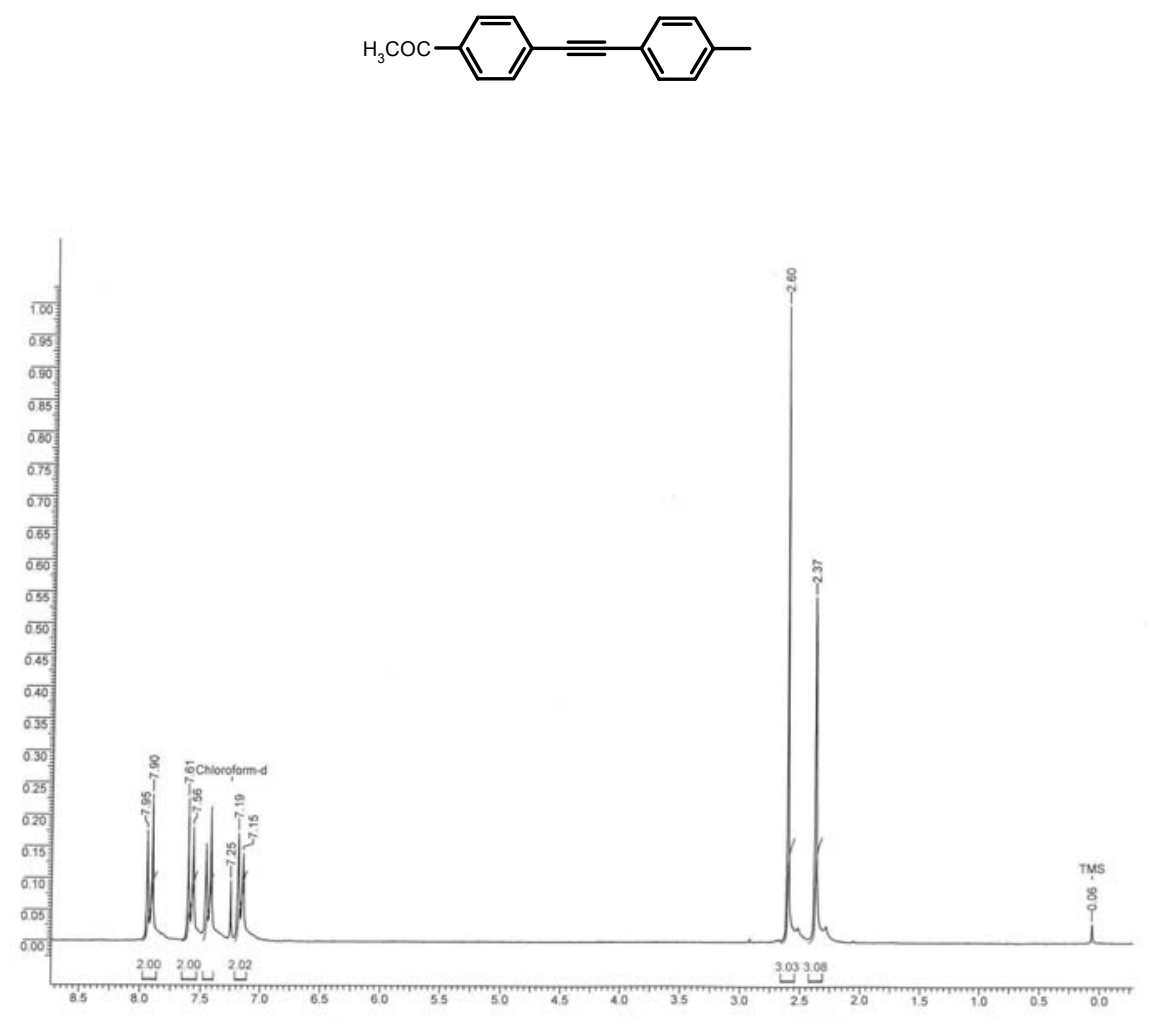

Aromatic region (Entry 19)

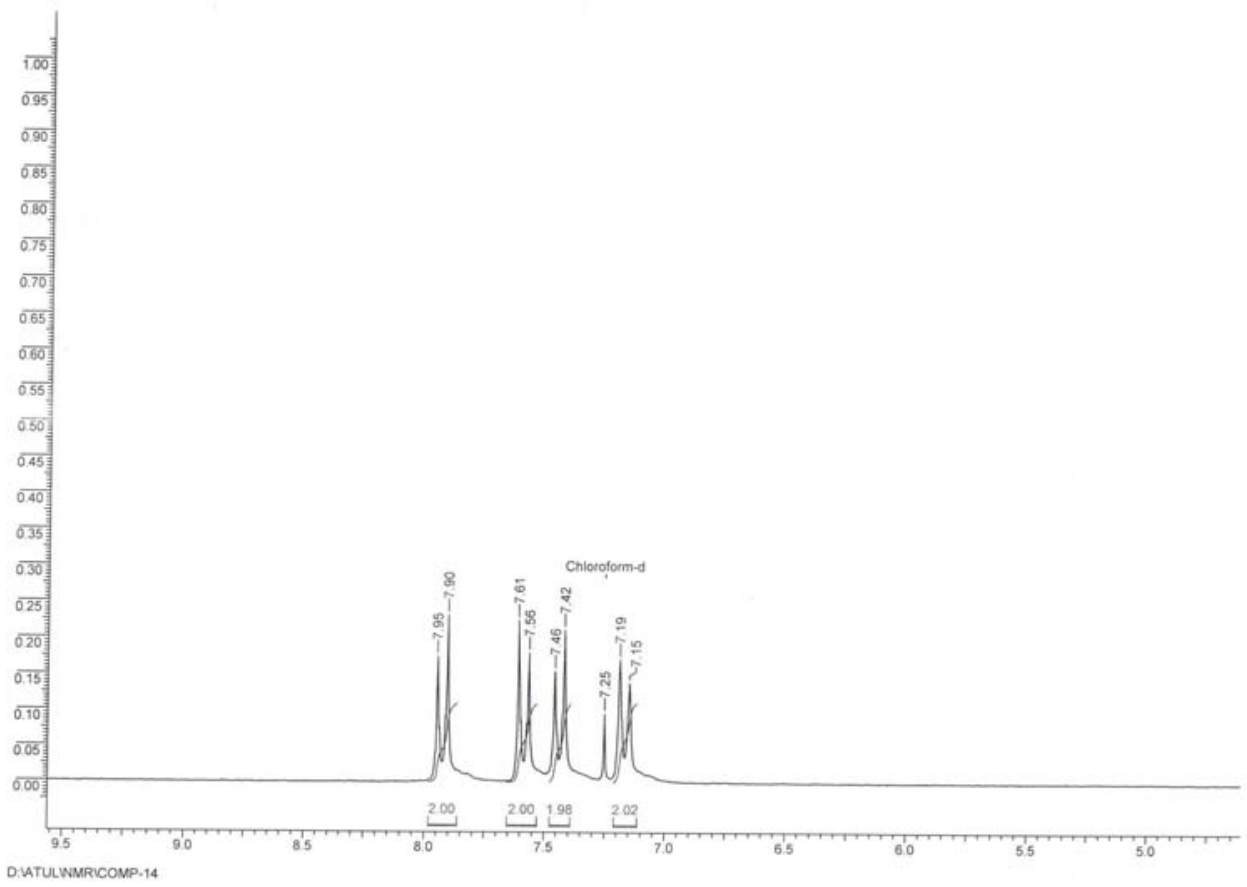



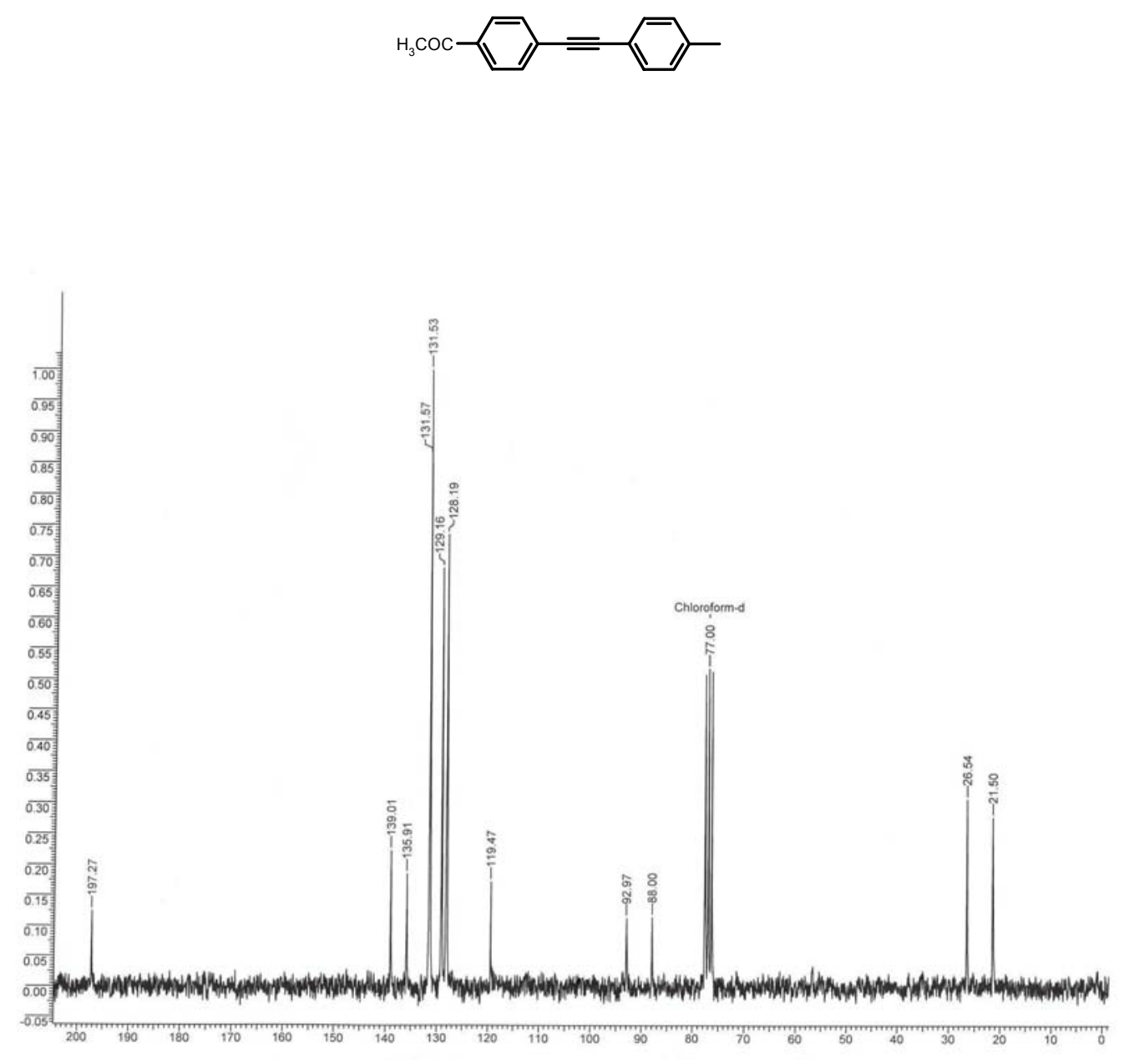

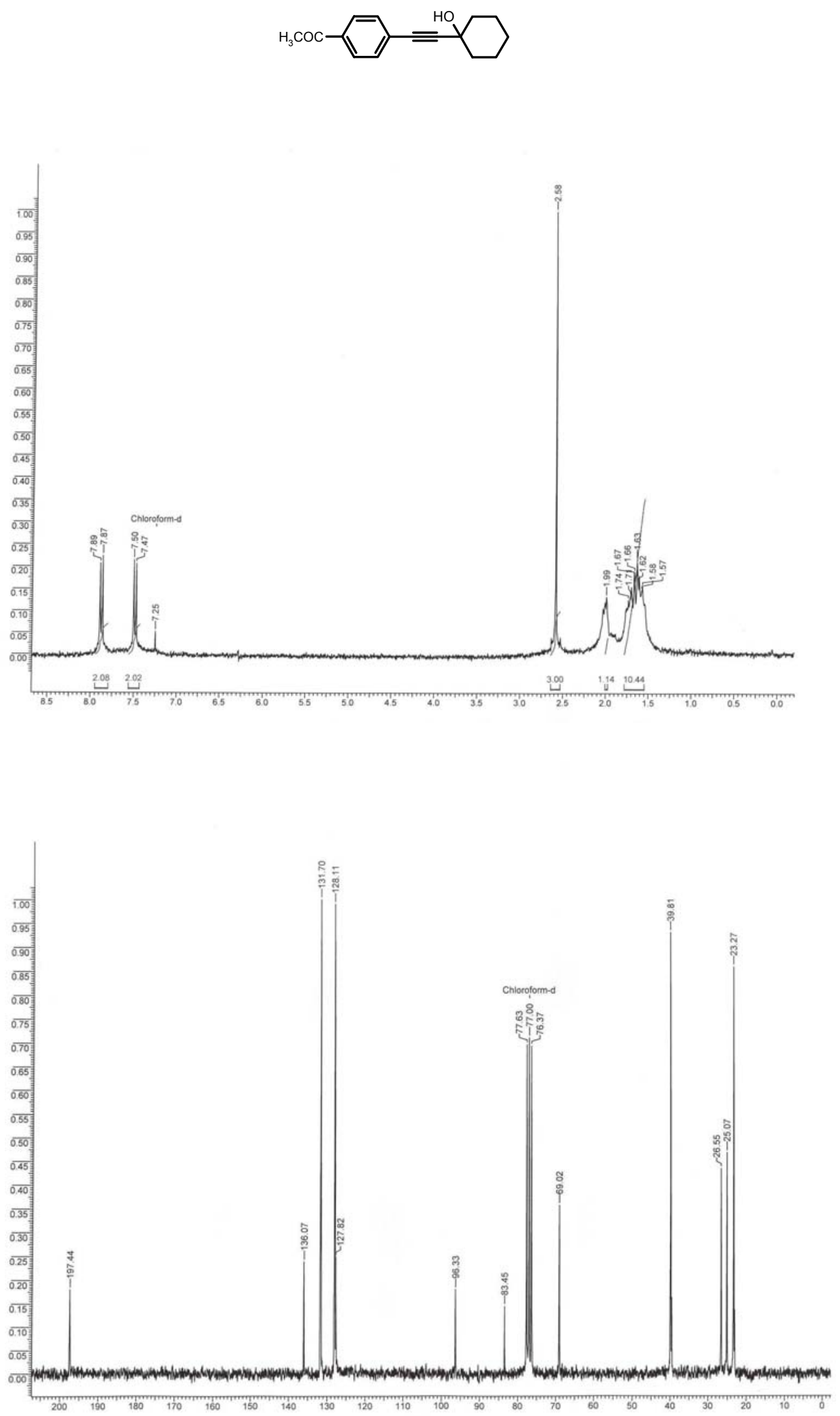

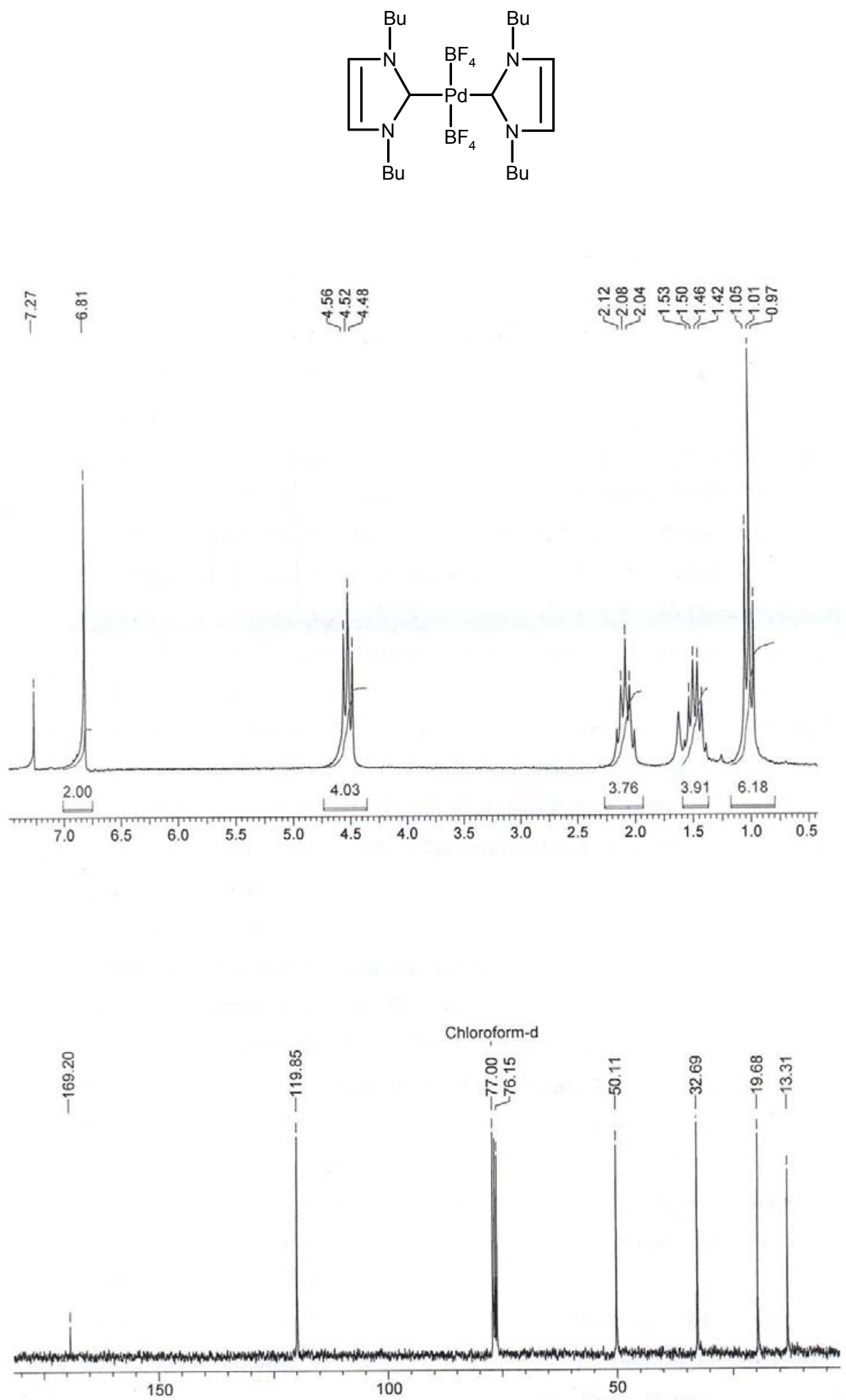


\section{References for known compounds:}

(1) Palimkar, S. S.; Siddiqui, S. A.; Daniel, T.; Lahoti, R. J.; Srinivasan, K. V. J. Org. Chem. 2003, 68, 9371.

(2) Ma, Y.; Song, C.; Jiang, W.; Wu, Q.; Wang, Y.; Liu, X.; Andrus, M. B. Org. Lett. 2003, $5,3317$.

(3) Michael R. E.; Zhaohui W., and Craig M. J.; Chem Commun. 2002, 818.

(4) Ishikawa, T.; Mizuta, T.; Hagiwara, K.; Aikawa, T.; Kudo, Takayuki; Saito, S. J. Org. Chem. 2003, 68, 3702 .

(5) Mio, M.J.; Kopel, K.C; Braun, J.B.; Gadzikwa, T.L.; Hull, K.L.; Brisbois, R.G.; Markworth, C.J.; Grieco, P.A. Org. Lett. 2002, 4, 3199.

(6) Okuro, K.; Furuune, M.; Enna, M.; Miura, M.; Nomura, M. J. Org. Chem. 1993, 58, 4716.

(7) Faller, J. W.; Kultyshev, R.G. Organometallics 2002, 21, 5911.

(8) Vasil'ev, A.V.; Rudenko, A. P. Russ. J. Org. Chem. 1997, 33, 1555.

(9) Deshmukh, R.R.; Rajagopal, R.; Srinivasan, K.V. Chem Commun. 2001, 1544. 